ALEA, Lat. Am. J. Probab. Math. Stat. 17, 329-353 (2020)

DOI: 10.30757/ALEA.v17-13

\title{
Anisotropic Gaussian wave models
}

\author{
Anne Estrade and Julie Fournier \\ MAP5 - UMR CNRS 8145 \\ Université de Paris \\ France. \\ E-mail address: anne.estrade@parisdescartes.fr \\ Affiliation of Second Author \\ Université Paris-Saclay \\ France. \\ E-mail address: julie.fournier@universite-paris-saclay.fr
}

\begin{abstract}
Let $d$ be an integer greater or equal to 2 and let $\mathbf{k}$ be a $d$-dimensional random vector. We call Gaussian wave model with random wavevector $\mathbf{k}$ any stationary Gaussian random field defined on $\mathbb{R}^{d}$ with covariance function $t \mapsto$ $\mathbb{E}[\cos (\mathbf{k} . t)]$. Any stationary Gaussian random field on $\mathbb{R}^{d}$ can be studied as a random wave. The purpose of the present paper is to link properties of the random wave with the distribution of the random wavevector, with a focus on geometric properties. We mainly concentrate on the study of the level sets measure, giving for instance a local estimate of its variance. In the planar case, we prove that the expected length of the nodal lines is decreasing as the anisotropy of the wavevector is increasing, and we study the direction that maximizes the expected length of the crest lines. We illustrate our results on two specific models: a generalization of Berry's monochromatic planar waves and a spatiotemporal sea wave model whose random wavevector is supported by the Airy surface in $\mathbb{R}^{3}$. According to a general theorem, these two Gaussian fields are anisotropic almost sure solutions of partial differential equations that involve the Laplacian operator: $\Delta f+\kappa^{2} f=0$ (where $\kappa=\|\mathbf{k}\|)$ for the former, $\Delta f+\partial_{t}^{4} f=0$ for the latter.
\end{abstract}

\section{Introduction}

For many centuries, physicists have been using wave models defined on a multidimensional space in various domains as different as acoustics, electronics, geophysics, oceanography or seismology. In order to take into account variability or

Received by the editors May 24th, 2019; accepted February 14th, 2020

2010 Mathematics Subject Classification. primary 60G60; secondary 60G15, 60K40, 62H11, $86 \mathrm{~A} 05$.

Key words and phrases. Gaussian field; random wave; nodal statistics; level set; crossing theory; anisotropy. 
uncertainty, it is useful to consider random wave models. It is the exact purpose of a pioneer exhaustive study by Longuet-Higgins (1957) that was concerned with sea waves modelized as a random moving surface. Another mathematical pioneer study was raised by Berry in several papers, Berry (1977) or Berry and Dennis (2000) for instance. These seminal works opened a wide area of research in the last decades, either for statistical purposes (Azaïs et al., 2005, Lindgren, 2006, Aberg et al., 2008, Azaïs et al., 2011, Beliaev et al., 2019, Nourdin et al., 2019), or more recently for topological purposes in link with number theory (Rudnick and Wigman, 2008, Krishnapur et al., 2013, Marinucci et al., 2016). Ten years ago, the interest for nodal sets or level sets also met the theory of crossings developed by Rice for one-dimensional stochastic processes fifty years before, yielding two inspiring books by Adler and Taylor (2007) and by Azaïs and Wschebor (2009). The present paper is clearly inspired by all the above references but to the best of our knowledge it is the first time that the different models are gathered in the same work and are studied under the same focus: anisotropy.

A big demand for anisotropic models is nowadays observed, in particular by practitioners in geostatistics, offshore engineering, heterogeneous material or medical imaging (see for instance Richard and Biermé, 2010, Klatt, 2016, Allard et al., 2016), but also for more theoretical studies dedicated to image synthesis and analysis, optics, cosmology or arithmetic (Biermé et al., 2015, Olhede et al., 2014, De Angelis et al., 2016, Ade et al., 2016, Kurlberg and Wigman, 2017).

In the present paper, we aim at exploring the anisotropy of stationary random fields that are defined on a $d$-dimensional space with $d \geq 2$. The random fields that we consider are stationary centred Gaussian fields whose covariance function is given by $t \in \mathbb{R}^{d} \mapsto \mathbb{E}[\cos (\mathbf{k} \cdot t)]$ for any $d$-dimensional random vector $\mathbf{k}$. We call them random waves since each of them has the same covariance function as any single random wave prescribed by $t \in \mathbb{R}^{d} \mapsto \sqrt{2} \cos (\mathbf{k} \cdot t+\eta)$, where $\eta$ is uniformly distributed on $[0,2 \pi]$ and independent from $\mathbf{k}$. Let us stress that the class of Gaussian random waves actually coincides with the one of stationary Gaussian fields that are centred and have unit variance. Indeed, for any such a field, it is sufficient to consider any random vector $\mathbf{k}$, called random wavevector, whose distribution is equal to its spectral measure. Our purpose is to link the geometric and anisotropic behaviour properties of any Gaussian random wave with the distribution of its random wavevector.

We like to mention that when the random wavevector $\mathbf{k}$ is equal to $A \mathbf{u}$ with $A$ a deterministic matrix and $\mathbf{u}$ a random vector in $\mathbb{R}^{d}$ whose distribution is invariant under rotations, the associated Gaussian random wave has the same distribution as an isotropic random wave deformed by the linear transformation $A^{T}$. In that case, the study of anisotropy, either in the spectral domain, or in the parameter domain, is equivalent. In the general case where no linear deformation is involved, studying anisotropy in those two domains falls under two different approaches. The latter point of view is adopted in Allard et al. (2016) or in Fournier (2018) for instance, whereas our paper definitively belongs to the former type as did Bonami and Estrade (2003) or Richard and Biermé (2010).

In order to illustrate our results, some models are specified. A famous case is Berry's monochromatic random waves model (see Berry, 1977 as a precursor or Berry, 2002; Beliaev et al., 2019; Nourdin et al., 2019 for recent studies in a twodimensional context), which is the isotropic solution of Helmholtz equation and is 
prescribed by a random wavevector uniformly distributed on a sphere. The planar version of Berry's monochromatic random waves has been extensively studied in the last decade for the outstanding behaviour of its nodal length variance in the highenergy limit. Although interested in the nodal sets, our work does not focus on this particular point. Actually, we go beyond Berry's model and we exhibit anisotropic solutions of Helmholtz equation that we call anisotropic Berry's monochromatic random waves. Another famous model is studied in the present paper: a spacetime model adjusted for sea waves (see Longuet-Higgins, 1957). It is a Gaussian random wave indexed by $\mathbb{R}^{2} \times \mathbb{R}$ that indicates the sea height at each point and each time instant. Its three-dimensional random wavevector is forced to live in a two-dimensional surface known as Airy surface. All over the paper, two specific distributions for the random wavevector in dimension two are examined. One is called "elementary model". It is described by a main direction and a bandwidth that quantifies the anisotropy. We call the other one "toy model". It is given by a positive probability density function only depending on a single parameter that carries out the whole quantified information on anisotropy.

Our major contribution to the study of anisotropic Gaussian random fields is twofold: qualitative with a new point of view based on the associated wavevector, and quantitative with closed formulas linking the features of a random wave with the features of its wavevector. Concerning the former, we give a necessar and sufficient condition on the wavevector for the random wave to solve a partial differential equation almost surely (see Theorem 2.5). Concerning the latter, we give an upperbound of the variance of the nodal sets measure when restricted to a small ball in $\mathbb{R}^{d}$ (see Proposition 3.2) and we prove that the expected length of the nodal lines of planar random waves is a decreasing function of the anisotropy of its random wavevector (see Proposition 3.4). We extend our study by presenting a formula for the expected length of a crest, which is the locus of points whose gradient has a prescribed direction (see Proposition 4.3). It allows us to prove that the direction that maximises the expected length of the crest lines is not necessarily orthogonal to the mode of the random wavevector's direction. For this purpose, we properly quantify anisotropy through various concepts, such as coherency index or favorite direction which are borrowed from spatial directional statistics.

The paper is organised as follows. General facts are presented in Section 2, in particular the key point of spectral representation, the link with partial differential equations that are solved by the random waves and at last the presentation of various models of Gaussian random waves characterized by the distribution of their random wavevector. In Section 3, we focus on the nodal sets, their Hausdorff measure (with an expectation formula and an upper-bound on the variance) and their directional statistics. Section 4 is devoted to the study of crest lines and their expected length, in the planar case. The technical computations are detailed in the Appendix section.

Notations and definitions. We write $\mathbb{N}_{0}$ for the set $\{0,1,2, \cdots\}$ of the nonnegative integers and $\mathbb{Z}_{+}$the set of positive integers.

Let an integer $d \geq 2$. We fix an orthonormal basis of $\mathbb{R}^{d}$ and we use the same notation for a vector $z$ in $\mathbb{R}^{d}$ and the vector of its coordinates in this basis. For any $z$ and $z^{\prime}$ in $\mathbb{R}^{d}$, we write $z \cdot z^{\prime}$ the canonical Euclidian scalar product of $z$ and $z^{\prime},\|\cdot\|$ the associated norm and $I_{d}$ the identity matrix of size $d$. For $\varphi \in[0,2 \pi]$, $u_{\varphi}$ denotes the vector $(\cos \varphi, \sin \varphi)$ in $\mathbb{R}^{2}$. 
For $\mathbf{j}=\left(j_{1}, \cdots, j_{d}\right) \in \mathbb{N}_{0}{ }^{d}$, we write $|\mathbf{j}|=\sum_{l=1}^{d} j_{l}$. Moreover, if $\lambda \in \mathbb{R}^{d}$ and if $F$ is a smooth map from $\mathbb{R}^{d}$ to $\mathbb{R}$, we write

$$
\lambda^{\mathbf{j}}=\prod_{l=1}^{d} \lambda_{l}^{j_{l}} \text { and } \partial^{\mathbf{j}} F=\frac{\partial^{|\mathbf{j}|} F}{\partial^{j_{1}} \lambda_{1} \cdots \partial^{j_{d}} \lambda_{d}} .
$$

We also denote by $F^{\prime}(t)$ and by $F^{\prime \prime}(t)$ the gradient vector and the Hessian matrix of $F$ at point $t$, respectively.

For any positive integer $s, \mathcal{H}_{s}$ denotes the Hausdorff measure of dimension $s$.

Let $Z=\left(z_{i}\right)_{1 \leq i \leq d}$ be a random vector in $\mathbb{R}^{d}$. We write $\mathbb{E}[Z]$ its $d$-dimensional expectation vector, $Z Z^{T}$ the $d \times d$ matrix $\left(z_{i} z_{j}\right)_{1 \leq i, j \leq d}$ and $\mathbb{E}\left[Z Z^{T}\right]$ the matrix of the second moments of $Z$. The standard Gaussian probability density function on $\mathbb{R}^{d}$ is denoted by $\Phi_{d}$.

Let $X$ be a real Gaussian random field defined on $\mathbb{R}^{d}$. It is said to be stationary if for any translation $\tau$ of $\mathbb{R}^{d}, X \circ \tau$ and $X$ have the same law. Then we can define its covariance function $r$ on $\mathbb{R}^{d}$ such that

$$
\forall t \in \mathbb{R}^{d}, \quad r(t)=\operatorname{Cov}(X(t), X(0)) .
$$

Furthermore, $X$ is said to be isotropic if for any rotation $\rho$ in $\mathbb{R}^{d}$ (element of the special orthogonal group $S 0(d)), X \circ \rho$ and $X$ have the same law, which is equivalent (if $X$ is also stationary) to $r$ being radial, that is to $r(t)$ depending on $t$ only through $\|t\|$.

\section{Anisotropic Gaussian wave model}

2.1. Definition. If $X: \mathbb{R}^{d} \rightarrow \mathbb{R}$ is a centred and stationary Gaussian random field with unit variance, according to Bochner's theorem, there exists a symmetric probability measure $F$ on $\mathbb{R}^{d}$ and a symmetric random variable $\mathbf{k}$ in $\mathbb{R}^{d}$ (with probability measure $F$ ), such that the covariance function $r$ of $X$ is given by

$$
\forall t \in \mathbb{R}^{d}, \quad r(t)=\mathbb{E}[X(0) X(t)]=\int_{\mathbb{R}^{d}} \exp (i u \cdot t) d F(u)=\mathbb{E}[\cos (\mathbf{k} \cdot t)] .
$$

Note that the distribution of $\mathbf{k}$ satisfying (2.1) for a given Gaussian field $X$ is no more unique if one drops the symmetry assumption. In the following, we will call random wavevector associated with $X$ any random vector $\mathbf{k}$ in $\mathbb{R}^{d}$ that satisfies (2.1).

Besides, let us quote that for any random vector $\mathbf{k}$, the map $t \mapsto \mathbb{E}[\cos (\mathbf{k} \cdot t)]$ actually defines a covariance function. Indeed, the single random wave $(\sqrt{2} \cos (\mathbf{k}$. $t+\eta))_{t \in \mathbb{R}^{d}}$, where $\eta$ is uniformly distributed in $[0,2 \pi]$, defines a stationary centred random field with the prescribed covariance function. Therefore, we introduce the next definition.

Definition 2.1. Let $\mathbf{k}$ be a $d$-dimensional random vector. We define $G_{\mathbf{k}}$, the Gaussian random wave associated with $\mathbf{k}$, as the real, centred, stationary and Gaussian random field on $\mathbb{R}^{d}$ with covariance function given by (2.1).

Due to Kolmogorov extension theorem (see Azaïs and Wschebor, 2009, Sections 1.1 and 1.2, for instance), such a field exists and its distribution is unique.

Let $\mathbf{k}$ be a $d$-dimensional random vector and let $G_{\mathbf{k}}$ be the Gaussian random wave associated with $\mathbf{k}$. Thanks to Equation (2.1), one can see that the spectral measure of $G_{\mathbf{k}}$ is equal to the probability measure of $\mathbf{k}^{s}$, the symmetrized random variable 
associated to $\mathbf{k}^{1}$. Hence, the random field $G_{\mathbf{k}}$ is, generally speaking, anisotropic and its anisotropy depends on the law of $\mathbf{k}$. In particular, $G_{\mathbf{k}}$ is isotropic if and only if the law of $\mathbf{k}^{s}$ is invariant under rotations.

Another consequence of the prescribed covariance function of $G_{\mathbf{k}}$ given by (2.1) is that $G_{\mathbf{k}}$ has unit variance. Moreover, the covariance function $r$ of $G_{\mathbf{k}}$ admits derivatives up to order $m\left(m \in \mathbb{N}_{0}\right)$ if and only if $\mathbf{k}$ admits moments of order $m$. In this case, for any $\mathbf{j} \in \mathbb{N}_{0}{ }^{d}$ such that $|\mathbf{j}| \leq m$, we have

$$
\partial^{\mathbf{j}} r(0)=0 \text { if }|\mathbf{j}| \text { is odd ; } \partial^{\mathbf{j}} r(0)=(-1)^{|\mathbf{j}| / 2} \mathbb{E}\left[\mathbf{k}^{\mathbf{j}}\right] \text { if }|\mathbf{j}| \text { is even. }
$$

In particular, $r^{\prime \prime}(0)=-\mathbb{E}\left[\mathbf{k k}^{T}\right]$. Besides, if the random wavevector $\mathbf{k}$ admits moments of any order, then $r$ is of class $\mathcal{C}^{\infty}$ and consequently there exists a version of $G_{\mathbf{k}}$ with almost every realization of $\operatorname{class} \mathcal{C}^{\infty}$.

In order to build a Gaussian field with the same distribution as $G_{\mathbf{k}}$, one way is to deduce a spectral representation from the integral expression (2.1) of its covariance function. To do so, let $W_{F}$ be a complex Gaussian $F$-noise on $\mathbb{R}^{d}$, i.e. a $\mathbb{C}$-valued process defined on the set $\mathcal{B}\left(\mathbb{R}^{d}\right)$ of Borelians such that

- a.s. $W_{F}$ is a complex-valued measure on $\mathcal{B}\left(\mathbb{R}^{d}\right)$,

- $\forall A \in \mathcal{B}\left(\mathbb{R}^{d}\right), W_{F}(A)$ is a complex-valued Gaussian variable with $\mathbb{E}\left[W_{F}(A)\right]=0$ and $\mathbb{E}\left[W_{F}(A) \overline{W_{F}(A)}\right]=F(A)$, where $\cdot$ denotes the complex conjugation,

- for any sequence $\left(A_{n}\right)_{n \in \mathbb{Z}_{+}}$of pairwise disjoint Borel sets, $\left(W_{F}\left(A_{n}\right)\right)_{n \in \mathbb{Z}_{+}}$ are independent random variables.

Moreover, we add the property that for any $A \in \mathcal{B}\left(\mathbb{R}^{d}\right), \overline{W_{F}(A)}=W_{F}(-A)$. Then, it is easy to check that the Gaussian stationary random field prescribed by

$$
\left(\int_{\mathbb{R}^{d}} e^{i t \cdot u} d W_{F}(u)\right)_{t \in \mathbb{R}^{d}}
$$

is real-valued, centred and that its covariance function is given by (2.1).

Starting from the random wavevector $\mathbf{k}$, we can also obtain an asymptotic representation for $G_{\mathbf{k}}$ as a limit of a sum of single random waves with the same covariance function. For this let us introduce identically distributed versions $\left(\mathbf{k}_{j}\right)_{j \in \mathbb{N}}$ of $\mathbf{k}$ and uniformly distributed random variables $\left(\eta_{j}\right)_{j \in \mathbb{Z}_{+}}$on $[0,2 \pi]$ and assume that all these random variables are independent. According to the central limit theorem applied to finite-dimensional distributions, the distribution of the random field

$$
\left(\sqrt{\frac{2}{N}} \sum_{j=1}^{N} \cos \left(\mathbf{k}_{j} \cdot t+\eta_{j}\right)\right)_{t \in \mathbb{R}^{d}}
$$

converges as $N$ tends to $\infty$ towards a Gaussian random field with covariance function $r$ given by (2.1).

\footnotetext{
${ }^{1}$ If $F_{\mathbf{k}}$ and $F_{-\mathbf{k}}$ are respectively the probability measures of $\mathbf{k}$ and $-\mathbf{k}$, then the symmetrized random variable associated with $\mathbf{k}$ is defined as the random variable with probability measure $F=\frac{1}{2}\left(F_{\mathbf{k}}+F_{-\mathbf{k}}\right)$.
} 
2.2. Random wavevector. In this paper, we focus on two families of distributions for $\mathbf{k}$. First, we are interested in cases where the random wavevector $\mathbf{k}$ is supported by the zero set of a multivariate polynomial $P,\left\{\lambda \in \mathbb{R}^{d}: P(\lambda)=0\right\}$. In particular, Section 2.3 deals with this assumption. Besides, we will also concentrate on the case where $\mathbf{k}$ is separable, in the sense of the following definition.

Definition 2.2. Let $\mathbf{k}$ be a random vector in $\mathbb{R}^{d}$. We say that $\mathbf{k}$ is separable if a.s. $\|\mathbf{k}\| \neq 0$ and if $\|\mathbf{k}\|$ and $\frac{1}{\|\mathbf{k}\|} \mathbf{k}$ are independent.

If $\mathbf{k}$ is separable, we write $\mathbf{k}=\|\mathbf{k}\| \tilde{\mathbf{k}}$, where $\tilde{\mathbf{k}}$ is a random variable in $\mathbb{S}^{d-1}$. For instance, if $\|\mathbf{k}\|$ is almost surely constant equal to $\kappa>0$, then $\mathbf{k}$ is separable and it is as well supported by the zero set of $P(x)=\sum_{i=1}^{d} x_{i}^{2}-\kappa^{2}$. In this case, we call $\kappa=\|\mathbf{k}\|$ the wavenumber of $G_{\mathbf{k}}$. In Section 2.4.1, we introduce properly this particular model corresponding to what we call anisotropic Berry's monochromatic random waves. We refer to Section 2.4 for specific examples of distributions of the random wavevectors.

If $\mathbf{k}$ is centred, which is for instance the case if $\mathbf{k}$ has a symmetric law, the coefficients of matrix $\mathbb{E}\left[\mathbf{k k}^{T}\right]$ are the covariances between the coordinates of $\mathbf{k}$ in our basis. In the planar case, this matrix is involved in the definition of the coherency index of $\mathbf{k}$.

Definition 2.3 (Coherency index). Let $\mathbf{k}$ be a random vector in $\mathbb{R}^{2}$. We assume that the symmetric non-negative matrix $\mathbb{E}\left[\mathbf{k k}^{T}\right]$ is non zero. We write $\lambda_{-}$and $\lambda_{+}$ its eigenvalues, such that $0 \leq \lambda_{-} \leq \lambda_{+}$and $\lambda_{+}>0$. The coherency index of $\mathbf{k}$ is a real number in $[0,1]$ defined as

$$
c(\mathbf{k})=\frac{\lambda_{+}-\lambda_{-}}{\lambda_{+}+\lambda_{-}} .
$$

Despite the fact that matrix $\mathbb{E}\left[\mathbf{k k}^{T}\right]$ depends on the choice of the basis, note that the coherency index does not depend on it. The coherency index is a common tool in spatial statistics and physics to characterize the anisotropy of a model, see Mardia and Jupp (2000) and Klatt (2016) for instance. In Polisano et al. (2020+), it is also computed for the so-named structure tensor in order to quantify the anisotropy of an anisotropic Gaussian self-similar planar field with stationary increments.

Let us consider two extreme cases. In the isotropic case, $\lambda_{-}=\lambda_{+}$and $c(\mathbf{k})=0$. On the contrary, if $\mathbf{k}$ is totally anisotropic in the sense that $\mathbf{k}$ is a.s. directed along a single deterministic direction, then $\lambda_{-}=0$ and $c(\mathbf{k})=1$. Note also that if we write $\mathbb{E}\left[\mathbf{k k}^{T}\right]=\left(\begin{array}{ll}m_{2,0} & m_{1,1} \\ m_{1,1} & m_{0,2}\end{array}\right)$, then

$$
\lambda_{ \pm}=\frac{1}{2}(T \pm \sqrt{\Delta}) \text { and } c(\mathbf{k})=\frac{\sqrt{\Delta}}{T},
$$

where $T=m_{2,0}+m_{0,2}$ and $\Delta=\left(m_{2,0}-m_{0,2}\right)^{2}+4 m_{1,1}^{2}$.

Remark 2.4. If $\mathbf{k}$ is separable then its coherency index only depends on the directional distribution of $\mathbf{k}$. Indeed, writing $\mathbf{k}=\|\mathbf{k}\| \widetilde{\mathbf{k}}$, we obtain $\mathbb{E}\left[\mathbf{k k}^{T}\right]=$ $\mathbb{E}\left[\|\mathbf{k}\|^{2}\right] \mathbb{E}\left[\tilde{\mathbf{k}} \tilde{\mathbf{k}}^{T}\right]$, so $c(\mathbf{k})=c(\widetilde{\mathbf{k}})$ and the coherency index of $\tilde{\mathbf{k}}$ is simply the difference between the eigenvalues of $\mathbb{E}\left[\tilde{\mathbf{k}} \tilde{\mathbf{k}}^{T}\right]$ because the trace of this matrix is equal to one. 
2.3. Link with partial differential equation. We are going to prove that $G_{\mathbf{k}}$ satisfies a specific partial differential equation if and only if the random wavevector $\mathbf{k}$ is supported by a specific hypersurface of $\mathbb{R}^{d}$. Let $P$ be an even $d$-multivariate polynomial. Then there exists a sequence of real numbers $\left(\alpha_{\mathbf{j}}\right)_{\mathbf{j} \in \mathbb{N}_{0}{ }^{d}}$ with only finitely many non-zero terms, such that

$$
\forall \lambda \in \mathbb{R}^{d}, \quad P(\lambda)=\sum_{\mathbf{j} \in \mathbb{N}_{0}{ }^{d} ;|\mathbf{j}| \text { even }} \alpha_{\mathbf{j}} \lambda^{\mathbf{j}} .
$$

We associate with $P$ the following differential operator:

$$
\mathcal{L}_{P}(X)=\sum_{\mathbf{j} \in \mathbb{N}_{0}{ }^{d} ;|\mathbf{j}| \text { even }}(-1)^{|\mathbf{j}| / 2} \alpha_{\mathbf{j}} \partial^{\mathbf{j}} X .
$$

Theorem 2.5. Let $P$ be an even multivariate polynomial defined by (2.4) and let $X$ be a Gaussian random field defined on $\mathbb{R}^{d}$ that is centred, stationary, with unit variance and almost surely of class $\mathcal{C}^{\infty}$. The following properties are equivalent.

(1) The Gaussian random field $X$ almost surely solves the partial differential equation $\mathcal{L}_{P}(X)=0$.

(2) Any random wavevector $\mathbf{k}$ associated with $X$ almost surely satisfies $P(\mathbf{k})=$ 0 .

(3) The covariance function $r$ of $X$ solves the partial differential equation $\mathcal{L}_{P}(r)=0$.

(4) The spectral measure $F$ of $X$ is supported by $\left\{\lambda \in \mathbb{R}^{d}: P(\lambda)=0\right\}$.

The above theorem allows us to exhibit random anisotropic solutions of some famous partial differential equations. Let us give an example, using the Laplacian operator $\Delta$ on $\mathbb{R}^{d}$, defined by $\Delta=\sum_{1 \leq j \leq d} \frac{\partial^{2}}{\partial t_{j}^{2}}$. If for some positive $\kappa, \mathbf{k} \in \kappa \mathbb{S}^{d-1}$, then $G_{\mathbf{k}}$ is an almost sure solution of Helmholtz equation $\Delta X+\kappa^{2} X=0$. In the same vein, the Gaussian random wave defined on $\mathbb{R}^{3}$, associated with a random wavevector with support in the Airy surface $\left\{(x, y, z) \in \mathbb{R}^{3} ; x^{2}+y^{2}-z^{4}=0\right\}$ is an almost sure solution of the partial differential equation $\frac{\partial^{2}}{\partial x^{2}} X+\frac{\partial^{2}}{\partial y^{2}} X+\frac{\partial^{4}}{\partial z^{4}} X=0$. It is used as a spatiotemporal random sea wave model, which is thoroughly presented in Section 2.4.3.

Proof of Theorem 2.5. Items 1. and 4. are equivalent as

$$
\begin{aligned}
\operatorname{Var}\left(\mathcal{L}_{P}(X)(t)\right) & =\operatorname{Var}\left(\mathcal{L}_{P}(X)(0)\right) \\
& =\sum_{\mathbf{j}, \mathbf{k} \in \mathbb{N}_{0}{ }^{d} ;|\mathbf{j}|,|\mathbf{k}| \text { even }}(-1)^{(|\mathbf{j}|+|\mathbf{k}|) / 2} \alpha_{\mathbf{j}} \alpha_{\mathbf{k}} \partial^{(\mathbf{j}+\mathbf{k})} r(0) \\
& =\int_{\mathbb{R}^{d}} P(\lambda)^{2} d F(\lambda) .
\end{aligned}
$$

Items 2. and 4. are equivalent as for any random wavevector $\mathbf{k}$ associated with $X$, and for $\mathbf{k}^{s}$ the symmetrized random variable associated to $\mathbf{k}$,

$$
\mathbb{E}[P(\mathbf{k})]=\mathbb{E}\left[P\left(\mathbf{k}^{s}\right)\right]=\int_{\mathbb{R}^{d}} P(\lambda)^{2} d F(\lambda) .
$$

At last, items 3. and 4. are equivalent as

$$
\mathcal{L}_{P}(r)(t)=\int_{\mathbb{R}^{d}} P(\lambda) e^{i \lambda \cdot t} d F(\lambda)=\widehat{P d F}(t),
$$

where we have denoted by $\hat{\cdot}$ the Fourier transform of any finite measure. 


\subsection{Presentation of the models.}

2.4.1. Anisotropic Berry's monochromatic waves. In this section, we focus on the case where the random wavevector $\mathbf{k}$ is such that, for some deterministic wavenumber $\kappa>0,\|\mathbf{k}\|=\kappa$ a.s., that is

$$
\kappa^{-1} \mathbf{k} \in \mathbb{S}^{d-1} \text { a.s.. }
$$

Since $\mathbf{k}$ is not necessarily isotropically distributed, the (unique in distribution) associated stationary centred Gaussian random field $G_{\mathbf{k}}$ on $\mathbb{R}^{d}$ is anisotropic. Since $\|\mathbf{k}\|$ is a.s. bounded, it is clear that $G_{\mathbf{k}}$ is a.s. of class $\mathcal{C}^{\infty}$. Hence, rephrasing Theorem 2.5, we get that $G_{\mathbf{k}}$ is the generic Gaussian solution of Helmholtz equation

$$
\Delta X+\kappa^{2} X=0
$$

Equivalently, $G_{\mathbf{k}}$ is an eigenfunction of the operator $-\Delta$, for the eigenvalue $\kappa^{2}$. Therefore, extending the definition introduced by Berry (1977) and intensively studied in the last years, we refer to $G_{\mathbf{k}}$ as an anisotropic Berry's monochromatic wave with random wavenumber $\kappa$.

Applying the change of variables $t \mapsto \kappa t$ yields the scaling property that $\left(G_{\mathbf{k}}(t)\right)_{t \in \mathbb{R}^{d}}$ and $\left(G_{\kappa^{-1} \mathbf{k}}(\kappa t)\right)_{t \in \mathbb{R}^{d}}$ have the same distribution, where we recall that the random vector $\kappa^{-1} \mathbf{k}$ takes its values in $\mathbb{S}^{d-1}$.

Besides, we remark that $\mathbf{k}^{s}$ is such that $\kappa^{-1} \mathbf{k}^{s}$ is supported by $\mathbb{S}^{d-1}$. Hence, we can deduce from Section 2.1 that the covariance function of $G_{\mathbf{k}}$ is given by

$$
r(t)=\int_{\mathbb{S}^{d-1}} e^{i \kappa u \cdot t} d \mu(u), \quad t \in \mathbb{R}^{d},
$$

where $\mu$ denotes the probability measure of $\kappa^{-1} \mathbf{k}^{s}$.

2.4.2. Planar and separable random waves. In this section, we set $d=2$ and we assume that the wavevector $\mathbf{k}$ is separable, in the sense of Definition 2.2. The two following examples of parametric distribution for the unitary wavevector $\tilde{\mathbf{k}}=$

$\frac{1}{\|k\|} \mathbf{k}$ will allow us to make computations to illustrate and comment the results of Sections 3 and 4 (see also Appendix Section A.1 for moments formulas). We write $\tilde{\mathbf{k}}=(\cos \Theta, \sin \Theta)$, where $\Theta$ is a random variable in $[0,2 \pi]$ and we fix $\theta_{0}$ in $[0,2 \pi]$.

Example 1 (Toy model) Let $\alpha \geq 0$. The density of $\Theta$ with respect to Lebesgue measure on $[0,2 \pi]$ is given by

$$
\theta \mapsto C_{\alpha}\left|\cos \left(\theta-\theta_{0}\right)\right|^{\alpha}, \text { with } C_{\alpha}=\frac{\Gamma(1+\alpha / 2)}{2 \sqrt{\pi} \Gamma(1 / 2+\alpha / 2)},
$$

where $\Gamma$ is the usual Gamma function. Parameter $\alpha$ is considered as an anisotropy parameter: $\alpha=0$ brings an isotropic model, whereas, at the opposite, $\alpha \rightarrow+\infty$ corresponds with a totally anisotropic random field since $\mathbf{k}$ is a.s. oriented along the $x$-axis. Our toy model is inspired by Klatt (2016) Section 2.1.2, where it is introduced to represent anisotropic spatial structures in physics. It is also used in De Angelis et al. (2016) with $\alpha=2$ and $\theta_{0}=0$ or $\theta_{0}=\pi / 2$ to modelize the two coordinates of a two-dimensional electromagnetic wave.

Example 2 (Elementary model) The random variable $\Theta$ is uniformly distributed on $\left[\theta_{0}-h, \theta_{0}+h\right]$ with $0 \leq h \leq \pi$. Parameter $\theta_{0}$ indicates the main direction whereas parameter $h$ negatively quantifies anisotropy, in the sense that 
the more anisotropic the model is, the smaller parameter $h$ is. Actually, $h=0$ corresponds with a totally anisotropic model, $h \approx 0$ corresponds with what is named narrow spectrum model in Longuet-Higgins (1957) Section I.6, and $h=\pi$ corresponds with the isotropic model. If one wishes a symmetric model, one can also consider $\Theta$ uniformly distributed on $\left[\theta_{0}-h, \theta_{0}+h\right] \cup\left[\theta_{0}+\pi-h, \theta_{0}+\pi+h\right]$ with $0 \leq h \leq \pi / 2$. The elementary model is studied in Biermé et al. (2015) and Polisano et al. $(2020+)$.

Up to a rotation of the basis, we can assume that in both examples $\theta_{0}=0$. Thus we set $\theta_{0}=0$ in the following.

2.4.3. Gaussian sea waves. In this section, we now concentrate on the case where the random wavevector $\mathbf{k}$ is 3 -dimensional and a.s. belongs to Airy surface, i.e.

$$
\mathbf{k} \in \mathcal{A}=\left\{\left(\lambda_{1}, \lambda_{2}, \lambda_{3}\right) \in \mathbb{R}^{3} ;\left(\lambda_{1}\right)^{2}+\left(\lambda_{2}\right)^{2}=\left(\lambda_{3}\right)^{4}\right\} \text { a.s.. }
$$

The covariance function of the Gaussian random wave $G_{\mathbf{k}}$ associated with $\mathbf{k}$ is

$$
r(t)=\mathbb{E}[\cos (\mathbf{k} \cdot t)]=\int_{\mathcal{A}} \cos (\lambda \cdot t) d F(\lambda), \quad t \in \mathbb{R}^{3},
$$

where $F$ is the probability distribution of $\mathbf{k}$ supported by $\mathcal{A}$.

The random field $G_{\mathbf{k}}$ coincides with the one used for the spatiotemporal random modelization of sea waves, assuming that the depth of the sea is infinite (see Longuet-Higgins, 1957 for the original idea, Azaiis et al., 2005 or Azaïs and Wschebor, 2009 for more recent developments). More precisely, for $(x, y, s) \in \mathbb{R}^{2} \times \mathbb{R}$, $G_{\mathbf{k}}(x, y, s)$ can be seen as the algebraic height of a wave at point $(x, y)$ and time $s$. If the moments of $\mathbf{k}$ are finite up to order four, we recall that according to Theorem $2.5, G_{\mathbf{k}}$ solves the partial differential equation

$$
\Delta G_{\mathbf{k}}+\partial_{t}^{4} G_{\mathbf{k}}=0
$$

with $\Delta$ the two-dimensional spatial Laplacian operator and $\partial_{t}^{4}$ the fourth temporal derivative.

We use the following parametrization of $\mathcal{A}$,

$$
(z, \theta) \in \mathbb{R} \times[0,2 \pi) \mapsto\left(z^{2} \cos \theta, z^{2} \sin \theta, z\right),
$$

which provides a bijection $\phi$ from $\mathbb{R} \backslash\{0\} \times[0,2 \pi)$ onto $\mathcal{A} \backslash\{(0,0,0)\}$. Performing the appropriate change of variables yields

$$
r(x, y, s)=\int_{\mathbb{R} \times(0,2 \pi)} \cos \left(x z^{2} \cos \theta+y z^{2} \sin \theta+s z\right) d F^{\phi}(z, \theta),
$$

where $F^{\phi}$ is the image of measure $F$ by the map $\phi^{-1}$. When $\mathbf{k}$ admits $f$ as probability density function with respect to the surface measure on $\mathcal{A}$, consequently to the coarea formula, we get

$$
r(x, y, s)=\int_{\mathbb{R} \times(0,2 \pi)} \cos \left(x z^{2} \cos \theta+y z^{2} \sin \theta+s z\right) g(z, \theta) d z d \theta,
$$

where the map $g$ is given by

$$
g(z, \theta)=f\left(z^{2} \cos \theta, z^{2} \sin \theta, z\right) z^{2}\left(1+4 z^{2}\right)^{1 / 2} .
$$

Following the literature, $g$ is called directional power spectrum of $G_{\mathbf{k}}$ (see Azaïs et al., 2005 and Azaïs and Wschebor, 2009 Chapter 11). Experimental directional 
power spectra are exhibited in Azaïs et al. (2005), derived from sea data provided by Ifremer.

Let us fix a time $s=s_{0}$ and look at the random field defined on $\mathbb{R}^{2}$,

$$
Z_{\mathbf{k}}(x, y)=G_{\mathbf{k}}\left(x, y, s_{0}\right) \quad(x, y) \in \mathbb{R}^{2},
$$

as a picture of the sea height at time $s_{0}$. It is a two-dimensional stationary centred Gaussian random field, whose covariance function is given by

$$
\begin{aligned}
r_{0}(x, y) & =r(x, y, 0) \\
& =\int_{\mathbb{R} \times(0,2 \pi)} \cos \left(x z^{2} \cos \theta+y z^{2} \sin \theta\right) d F^{\phi}(z, \theta) \\
& =\mathbb{E}[\cos ((x, y) \cdot \pi(\mathbf{k}))],
\end{aligned}
$$

where the random vector $\pi(\mathbf{k})$ is described in polar coordinates by $\left(R^{2}, \Theta\right)$ with $(R, \Theta)$ distributed according to measure $F^{\phi}$. Thus $\pi(\mathbf{k})$ is the random wavevector associated with $Z_{\mathbf{k}}$ and it is nothing but the projection of the $\mathcal{A}$-valued random wavevector $\mathbf{k}$ onto the plane of the first two coordinates. Consequently, the moments of $\pi(\mathbf{k})$ are given, for any integers $j$ and $k$ in $\mathbb{N}_{0}$, by

$$
m_{j, k}=\int_{(0,2 \pi) \times \mathbb{R}}\left(z^{2} \cos \theta\right)^{j}\left(z^{2} \sin \theta\right)^{k} d F^{\phi}(z, \theta),
$$

assuming that the above integral is finite.

Note that if $F^{\phi}$ can be written as a tensorial product measure: $d F^{\phi}(z, \theta)=$ $d \Xi(z) \otimes d \Lambda(\theta)$ then, according to Definition 2.2, $\pi(\mathbf{k})$ is separable.

\section{Level sets of Gaussian random waves}

Let $\mathbf{k}$ be a random wavevector in $\mathbb{R}^{d}$ admitting moments up to order four and let $G_{\mathbf{k}}$ be the associated Gaussian random field defined on $\mathbb{R}^{d}$. From now on in this section, we assume that $G_{\mathbf{k}}$ is almost surely of class $C^{2}$.

Let $a \in \mathbb{R}$. We are interested in the level set

$$
G_{\mathbf{k}}^{-1}(a)=\left\{t \in \mathbb{R}^{d}: G_{\mathbf{k}}(t)=a\right\},
$$

which is a.s. a $C^{2}$-submanifold of $\mathbb{R}^{d}$ with dimension $d-1$, called nodal set in the case $a=0$.

3.1. Expected measure. We are now interested in the expected measure of the level sets of $G_{\mathbf{k}}$. Let $Q$ be a compact set in $\mathbb{R}^{d}$ with non empty interior. We focus on the $(d-1)$-dimensional Hausdorff measure of the $a$-level set restricted to $Q$, namely

$$
\ell(a, \mathbf{k}, Q):=\mathcal{H}_{d-1}\left(G_{\mathbf{k}}^{-1}(a) \cap Q\right)=\mathcal{H}_{d-1}\left(\left\{t \in Q / G_{\mathbf{k}}(t)=a\right\}\right) .
$$

For now on in Section 3, we assume that $G_{\mathbf{k}}^{\prime}(0)$ is a non-degenerate Gaussian vector or, equivalently, that $\mathbb{E}\left[\mathbf{k k}^{T}\right]$ is invertible. This allows us to apply Kac-Rice formula (see Azaïs and Wschebor, 2009 Theorem 6.8 for instance). It yields

$$
\mathbb{E}[\ell(a, \mathbf{k}, Q)]=\int_{Q} \mathbb{E}\left[\left\|G_{\mathbf{k}}^{\prime}(t)\right\| \mid G_{\mathbf{k}}(t)=a\right] p_{G_{\mathbf{k}}(t)}(a) d t,
$$

where $p_{G_{\mathbf{k}}(t)}$, the probability density function of $G_{\mathbf{k}}(t)$, is actually given by the standard Gaussian distribution. Using the stationarity of $G_{\mathbf{k}}$ and the fact that for 
a fixed point $t, G_{\mathbf{k}}(t)$ and $G_{\mathbf{k}}^{\prime}(t)$ are independent random variables, we have

$$
\mathbb{E}[\ell(a, \mathbf{k}, Q)]=\mathcal{H}_{d}(Q) \frac{e^{-a^{2} / 2}}{\sqrt{2 \pi}} \mathbb{E}\left[\left\|G_{\mathbf{k}}^{\prime}(0)\right\|\right]
$$

Consequently, recalling that $\left\|G_{\mathbf{k}}^{\prime}(0)\right\|$ is the Euclidean norm of a $d$-dimensional centred Gaussian vector with variance matrix $-r^{\prime \prime}(0)=\mathbb{E}\left[\mathbf{k k}^{T}\right]$ and that $\Phi_{d}$ stands for the standard Gaussian probability density function on $\mathbb{R}^{d}$, we obtain

$$
\mathbb{E}[\ell(a, \mathbf{k}, Q)]=\mathcal{H}_{d}(Q) \frac{e^{-a^{2} / 2}}{\sqrt{2 \pi}} \int_{\mathbb{R}^{d}}\left(\mathbb{E}\left[\mathbf{k k}^{T}\right] x \cdot x\right)^{1 / 2} \Phi_{d}(x) d x
$$

In the separable case, we deduce from the above formula the following straightforward lemma.

Lemma 3.1. We assume that $\mathbf{k}$ is separable with $\mathbf{k}=\|\mathbf{k}\| \tilde{\mathbf{k}}$ and that $\mathbb{E}\left[\mathbf{k k}^{T}\right]$ is invertible. Then

$$
\mathbb{E}[\ell(a, \mathbf{k}, Q)]=\mathcal{H}_{d}(Q) \frac{e^{-a^{2} / 2}}{\sqrt{2 \pi}} \mathbb{E}\left[\|\mathbf{k}\|^{2}\right]^{1 / 2} \int_{\mathbb{R}^{d}}\left(\mathbb{E}\left[\widetilde{\mathbf{k}} \widetilde{\mathbf{k}}^{T}\right] x \cdot x\right)^{1 / 2} \Phi_{d}(x) d x
$$

The above formula applies to isotropic Berry's monochromatic random wave, i.e. to the case where $\|\mathbf{k}\|$ is a.s. constant equal to some positive constant $\kappa$ and $\widetilde{\mathbf{k}}$ is uniformly distributed in $\mathbb{S}^{d-1}$. In this case, $\mathbb{E}\left[\|\mathbf{k}\|^{2}\right]=\kappa^{2}$ and $\mathbb{E}\left[\tilde{\mathbf{k}} \tilde{\mathbf{k}}^{T}\right]=(1 / d) I_{d}$. Hence, the involved integral becomes $\int_{\mathbb{R}^{d}}\|x\| \Phi_{d}(x) d x$, which is the mean of a $\chi^{-}$ distributed random variable with $d$ degrees of freedom and is known to be equal to $\sqrt{2} \frac{\Gamma((d+1) / 2)}{\Gamma(d / 2)}$, with $\Gamma$ the usual Gamma function. Finally, we recover the well known formula expressing the expected measure of the $a$-level set of a Berry monochromatic random wave (see Berry, 1977 or Nourdin et al., 2019, Equation (1.11) in the planar case):

$$
\mathbb{E}[\ell(a, \mathbf{k}, Q)]=\mathcal{H}_{d}(Q) \frac{e^{-a^{2} / 2}}{\sqrt{d \pi}} \kappa \frac{\Gamma((d+1) / 2)}{\Gamma(d / 2)} .
$$

We complete the computation of the expected measure of nodal sets by giving an estimate of the variance. Actually, getting a closed formula for the variance seems a very difficult task. Whereas various studies are concerned with the asymptotic behaviour of the variance in the high-energy limit, or similarly as the compact domain $Q$ grows to $\mathbb{R}^{d}$, next proposition provides an upper-bound when the domain $Q$ is a shrinking ball $B(0, R)$ with $R$ going to 0 .

Proposition 3.2. For $R>0$ small enough,

$$
\operatorname{Var}[\ell(a, \mathbf{k}, B(0, R))] \leq C e^{-a^{2} / 2} R^{2 d-1} \frac{\mathbb{E}\left[\|\mathbf{k}\|^{2}\right]-\lambda_{\min }}{\lambda_{\text {min }}^{1 / 2}},
$$

where $\lambda_{\min }$ is the smallest eigenvalue of $\mathbb{E}\left[\mathbf{k k}^{T}\right]$ and $C$ is some universal positive constant.

Remark 3.3. In the above upper-bound, let us comment the factor $\left(\mathbb{E}\left[\|\mathbf{k}\|^{2}\right]-\right.$ $\left.\lambda_{\min }\right) \lambda_{\min }^{-1 / 2}$, depending on the covariance function of the random wave. In the separable case, writing $\tilde{\lambda}_{\text {min }}$ the smallest eigenvalue of $\mathbb{E}\left[\tilde{\mathbf{k}} \tilde{\mathbf{k}}^{T}\right]$, this factor can been expressed as $\left(\mathbb{E}\|\mathbf{k}\|^{2}\right)^{1 / 2}\left(1-\tilde{\lambda}_{\text {min }}\right) \tilde{\lambda}_{\text {min }}^{-1 / 2}$. 
In particular, for Berry's monochromatic random wave with wavenumber $\kappa$, we get for small radii $R$,

$$
\operatorname{Var}[\ell(a, \mathbf{k}, B(0, R))] \leq C e^{-a^{2} / 2} R^{2 d-1} \kappa \frac{1-\tilde{\lambda}_{m i n}}{\tilde{\lambda}_{m i n}^{1 / 2}}
$$

It should be compared with the asymptotic behaviour observed by Berry in the isotropic case (see Berry, 2002; Nourdin et al., 2019) when $d=2, a=0, R$ is fixed and $\kappa$ tends to infinity, which writes

$$
\operatorname{Var}[\ell(0, \mathbf{k}, B(0, R))] \sim C R^{2} \log (\kappa),
$$

for some universal constant $C>0$. Our upper-bound, being proportional to $R^{3}$, is clearly more acurate for small radii $R$. At the opposite, Berry's statement is sharper for big values of $\kappa$.

Proof: Let us start with Kac-Rice formula (see Azaïs and Wschebor, 2009, Theorem $6.9)$ to compute the second moment of $\ell(a, \mathbf{k}, Q)$ :

$$
\mathbb{E}\left[\ell(a, \mathbf{k}, Q)^{2}\right]=\int_{Q} \int_{Q} \mathbb{E}\left[\left\|G_{\mathbf{k}}^{\prime}(s)\right\|\left\|G_{\mathbf{k}}^{\prime}(t)\right\| \mid G_{\mathbf{k}}(s)=G_{\mathbf{k}}(t)=a\right] p_{s, t}(a, a) d s d t,
$$

where $p_{s, t}$ denotes the probability density function of $\left(G_{\mathbf{k}}(s), G_{\mathbf{k}}(t)\right)$. Using firstly the stationarity of $G_{\mathbf{k}}$ and secondly Cauchy-Schwarz inequality, we get

$$
\begin{aligned}
& \mathbb{E}\left[\ell(a, \mathbf{k}, Q)^{2}\right] \\
& =\int_{\mathbb{R}^{d}} \mathcal{H}_{d}(Q \cap Q-t) \mathbb{E}\left[\left\|G_{\mathbf{k}}^{\prime}(0)\right\|\left\|G_{\mathbf{k}}^{\prime}(t)\right\| \mid G_{\mathbf{k}}(0)=G_{\mathbf{k}}(t)=a\right] p_{0, t}(a, a) d t \\
& \leq \int_{\mathbb{R}^{d}} \mathcal{H}_{d}(Q \cap Q-t)\left(F_{a, \mathbf{k}}(t) F_{a, \mathbf{k}}(-t)\right)^{1 / 2} d t,
\end{aligned}
$$

where

$$
F_{a, \mathbf{k}}(t)=\mathbb{E}\left[\left\|G_{\mathbf{k}}^{\prime}(0)\right\|^{2} \mid G_{\mathbf{k}}(0)=G_{\mathbf{k}}(t)=a\right] p_{0, t}(a, a) .
$$

Let us now evaluate $F_{a, \mathbf{k}}(t)$. On the one hand, a straightforward regression shows that the conditional distribution of $G^{\prime}(0)$ given $\{G(0)=G(t)=a\}$ is Gaussian with mean $\frac{a}{1+r(t)} r^{\prime}(t)$ and covariance matrix $-r^{\prime \prime}(0)-\frac{1}{1-r(t)^{2}} r^{\prime}(t) r^{\prime}(t)^{T}$. On the other hand, if $Z$ is a Gaussian vector $\mathcal{N}(b, \Sigma)$, then $\mathbb{E}\left[\|Z\|^{2}\right]=\|b\|^{2}+\operatorname{Tr}(\Sigma)$ with $\operatorname{Tr}(\cdot)$ denoting the trace. Hence, since $\operatorname{Tr}\left(r^{\prime}(t) r^{\prime}(t)^{T}\right)=\left\|r^{\prime}(t)\right\|^{2}$, we get

$$
F_{a, \mathbf{k}}(t)=\left(\left(\frac{a}{1+r(t)}\right)^{2}\left\|r^{\prime}(t)\right\|^{2}-\operatorname{Tr}\left(r^{\prime \prime}(0)\right)-\frac{1}{1-r(t)^{2}}\left\|r^{\prime}(t)\right\|^{2}\right) \frac{e^{-a^{2} /(1+r(t))}}{2 \pi\left(1-r(t)^{2}\right)^{1 / 2}} .
$$

This formula shows that $F_{a, \mathbf{k}}(t)=F_{a, \mathbf{k}}(-t)$ and it puts us in position to write a Taylor expansion of $F_{a, \mathbf{k}}(t)$ as $\|t\|$ goes to 0 . In order to simplify computations, and without loss of generality, one can assume that an orthonormal basis of $\mathbb{R}^{d}$ is given such that the Hessian matrix $-r^{\prime \prime}(0)$ is diagonal, with eigenvalues $\lambda_{1} \geq \cdots \geq$ $\lambda_{d}>0$. Then, as $\|t\|$ goes to 0 ,

$$
r(t)=1-\frac{1}{2} \sum_{1 \leq i \leq d} \lambda_{i} t_{i}^{2}+o\left(\|t\|^{3}\right) \text { and }\left\|r^{\prime}(t)\right\|^{2}=\sum_{1 \leq i \leq d} \lambda_{i}^{2} t_{i}^{2}+o\left(\|t\|^{2}\right) .
$$


Hence,

$$
\begin{aligned}
F_{a, \mathbf{k}}(t) & =\left(\sum_{1 \leq i \leq d} \lambda_{i}-\frac{\sum_{1 \leq i \leq d} \lambda_{i}^{2} t_{i}^{2}}{\sum_{1 \leq i \leq d} \lambda_{i} t_{i}^{2}}\right) \frac{e^{-a^{2} / 2}}{2 \pi\left(\sum_{1 \leq i \leq d} \lambda_{i} t_{i}^{2}\right)^{1 / 2}}+o(\|t\|) \\
& =e^{-a^{2} / 2} \frac{\sum_{1 \leq i \leq d} \lambda_{i}\left(\sum_{j \neq i} \lambda_{j}\right) t_{i}^{2}}{2 \pi\left(\sum_{1 \leq i \leq d} \lambda_{i} t_{i}^{2}\right)^{3 / 2}}+o(\|t\|) \\
& \leq e^{-a^{2} / 2} \frac{\sum_{j \neq d} \lambda_{j}}{2 \pi \lambda_{d}^{1 / 2}}\|t\|^{-1}+o(\|t\|) .
\end{aligned}
$$

Now we integrate the latter upper bound of $F_{a, \mathbf{k}}(t)$ on the set of points $t$ such that $B(0, R) \cap B(0, R)-t \neq \emptyset$, which is equal to $B(0,2 R)$. It yields

$$
\mathbb{E}\left[\ell(a, \mathbf{k}, B(0, R))^{2}\right] \leq C e^{-a^{2} / 2} \frac{\sum_{j \neq d} \lambda_{j}}{\lambda_{d}^{1 / 2}} R^{d+d-1}=C e^{-a^{2} / 2} \frac{\mathbb{E}\left[\|\mathbf{k}\|^{2}\right]-\lambda_{d}}{\lambda_{d}^{1 / 2}} R^{2 d-1},
$$

since $\mathbb{E}\left[\|\mathbf{k}\|^{2}\right]=-\operatorname{Tr}\left(r^{\prime \prime}(0)\right)=\sum_{1 \leq i \leq d} \lambda_{i}$. As $\mathbb{E}[\ell(a, \mathbf{k}, B(0, R))]^{2}$ has order of magnitude $R^{2 d}$, it is negligible with respect to the second moment, and we get the announced upper bound for the variance of $\ell(a, \mathbf{k}, B(0, R))$.

3.2. Planar case. In the planar case, i.e. $d=2$, the level sets $G_{\mathbf{k}}^{-1}(a)$ are onedimensional. Furthermore assuming that $\mathbf{k}$ is separable, we will establish that the level curves mean length decreases with anisotropy. Our formula involves the coherency index, introduced in Definition 2.3.

To compute the integral in the right-hand side of (3.1), we use the following well known fact, that can be proved with simple algebra.

If $M$ is a symmetric positive definite matrix with eigenvalues $\gamma_{-}$and $\gamma_{+}$such that $0<\gamma_{-} \leq \gamma_{+}$, then

$$
\int_{\mathbb{R}^{2}}(M x \cdot x)^{1 / 2} \Phi_{2}(x) d x=\left(\frac{2 \gamma_{+}}{\pi}\right)^{1 / 2} \mathcal{E}\left(\left(1-\gamma_{-} / \gamma_{+}\right)^{1 / 2}\right)
$$

where $\mathcal{E}$ stands for the elliptic integral given by $\mathcal{E}(x)=\int_{0}^{\pi / 2}\left(1-x^{2} \sin ^{2} \theta\right)^{1 / 2} d \theta$, for $x \in[0,1]$.

In our case, we set $M=\mathbb{E}\left[\widetilde{\mathbf{k}} \widetilde{\mathbf{k}}^{T}\right]$ and $\gamma_{-}+\gamma_{+}=1$ since $\widetilde{\mathbf{k}}$ belongs to $\mathbb{S}^{1}$, a.s.. Hence, writing $c=c(\widetilde{\mathbf{k}})$, we have $2 \gamma_{+}=1+c$ and $1-\gamma_{-} / \gamma_{+}=\frac{2 c}{1+c}$. Consequently, the following proposition holds.

Proposition 3.4. Let $\mathbf{k}$ be a separable random wavevector in $\mathbb{R}^{2}$ such that $\mathbf{k}=$ $\|\mathbf{k}\| \widetilde{\mathbf{k}}$ and $\mathbb{E}\left[\mathbf{k k}^{T}\right]$ is invertible. Let us denote by $c(\widetilde{\mathbf{k}})=c(\mathbf{k})$ the coherency index of k. Then,

$$
\mathbb{E}[\ell(a, \mathbf{k}, Q)]=\mathcal{H}_{2}(Q) \frac{e^{-a^{2} / 2}}{\pi \sqrt{2}} \mathbb{E}\left[\|\mathbf{k}\|^{2}\right]^{1 / 2} \mathcal{F}(c(\widetilde{\mathbf{k}})),
$$

where the map $\mathcal{F}: c \in[0,1] \mapsto(1+c)^{1 / 2} \mathcal{E}\left(\left(\frac{2 c}{1+c}\right)^{1 / 2}\right)$ is strictly decreasing.

The proof of the decrease of mapping $\mathcal{F}$ is postponed to the Appendix section, see Lemma A.3. Another expression for the same expectation can be found in Longuet-Higgins (1957) Formula (2.3.13), however our formulation highlights the effect of the wavevector's distribution on the mean length of level sets. 
Remark 3.5. Regarding the coherency index $c(\widetilde{\mathbf{k}})$ as a parameter that positively quantifies anisotropy, the above formula clearly indicates that the mean length of level curves is decreasing as the anisotropy of $\mathbf{k}$ increases.

Remark 3.6. In the planar case, the proof of Proposition 3.2 allows us to obtain a better upper bound for the variance of $\ell(a, \mathbf{k}, B(0, R))$, if $R$ is small enough, than the one stated in the proposition itself. To begin with, when $d=2$, Equality (3.2) can be written

$$
F_{a, \mathbf{k}}(t)=\frac{\lambda_{-}}{\lambda_{+}^{1 / 2}} \frac{\|t\|^{2}}{\left(t_{1}^{2}+\frac{\lambda_{-}}{\lambda_{+}} t_{2}^{2}\right)^{3 / 2}}+o(\|t\|) .
$$

We introduce another elliptic integral $\tilde{\mathcal{E}}(x)=\int_{0}^{\pi / 2}\left(1-x^{2} \sin ^{2} \theta\right)^{-3 / 2} d \theta$, for $x \in[0,1]$ and

$$
\mathcal{G}: c \in[0,1] \mapsto \frac{1-c}{(1+c)^{1 / 2}} \tilde{\mathcal{E}}\left(\left(\frac{2 c}{1+c}\right)^{1 / 2}\right) .
$$

Then, after integrating (3.4) in polar coordinates, we obtain

$$
\operatorname{Var}[\ell(a, \mathbf{k}, B(0, R))] \leq C^{\prime} e^{-a^{2} / 2} R^{3} \mathbb{E}\left[\|\mathbf{k}\|^{2}\right]^{1 / 2} \mathcal{G}(c(\widetilde{\mathbf{k}})),
$$

where $C^{\prime}$ is a universal constant.

We now apply Proposition 3.4 to our separable examples, prescribing the directional distribution of the wavevector $\mathbf{k}$. We refer to the Appendix Section A.1 for the detailed computation of their moments.

Example 1 (Toy model) Take $\widetilde{\mathbf{k}}$ distributed on $\mathbb{S}^{1}$ with probability density function given by $(2.5)$ for some positive $\alpha$. Then, $\mathbb{E}\left[\widetilde{\mathbf{k}} \widetilde{\mathbf{k}}^{T}\right]=\frac{1}{\alpha+2}\left(\begin{array}{cc}\alpha+1 & 0 \\ 0 & 1\end{array}\right)$ and consequently, $c(\widetilde{\mathbf{k}})=\frac{\alpha}{\alpha+2}$, which is an increasing function of parameter $\alpha$. As observed in Remark 3.5, the more anisotropic the model is, the smaller the expected length of level sets is.

Example 2 (Elementary model) We choose $\widetilde{\mathbf{k}}$ to be uniformly distributed on $[-h, h] \cup[\pi-h, \pi+h]$ for some $0<h \leq \pi / 2$. In that case, $\mathbb{E}\left[\widetilde{\mathbf{k}}^{T}\right]=$ $\frac{1}{2}\left(\begin{array}{cc}1+\frac{\sin (2 h)}{2 h} & 0 \\ 0 & 1-\frac{\sin (2 h)}{2 h}\end{array}\right)$. Hence, $c(\widetilde{\mathbf{k}})=\frac{\sin (2 h)}{2 h}$, which is decreasing on $(0, \pi / 2]$. Again, the mean length of level sets is decreasing with anisotropy, i.e. as $h$ is decreasing.

3.3. Favorite orientation. In this section, we introduce the directional statistics features of the random waves.

Definition 3.7. We call favorite direction of a random vector $V$ in $\mathbb{R}^{d}$ any $u \in \mathbb{S}^{d-1}$ that maximizes $\mathbb{E}\left[(V . u)^{2}\right]$.

Since $\mathbb{E}\left[(V . u)^{2}\right]=u . \mathbb{E}\left[V V^{T}\right] u$, the favorite directions of $V$ are the eigenvectors with norm one associated with the largest eigenvalue of the symmetric positive matrix $\mathbb{E}\left[V V^{T}\right]$. If the largest eigenvalue is simple, i.e. if the dimension of the associated eigenspace is one, then there are exactly two favorite directions, which are opposite one another. Note that if a random wavevector $\mathbf{k}$ is separable then its favorite directions are exactly the ones of $\tilde{\mathbf{k}}$. 
We turn to the directional study of the level set $G_{\mathbf{k}}^{-1}(a)$. For any $t \in G_{\mathbf{k}}^{-1}(a)$, the tangent space at point $t, T_{t} G_{\mathrm{k}}^{-1}(a)$, is a $(d-1)$-dimensional linear subspace that is orthogonal to the vector $G_{\mathbf{k}}^{\prime}(t)$. Using the previous definition, the favorite directions of $G_{\mathbf{k}}^{\prime}(t)$ are given by the unitary eigenvectors associated with the largest eigenvalue of $\mathbb{E}\left[G_{\mathbf{k}}^{\prime}(t) G_{\mathbf{k}}^{\prime}(t)^{T}\right]$. As the latter matrix is equal to $\mathbb{E}\left[\mathbf{k k}^{T}\right]$ (see Section 2.1), the favorite directions of $G_{\mathbf{k}}^{\prime}(t)$ coincide with those of $\mathbf{k}$. Hence, we get the next statement that sounds physically intuitive: the favorite orientations of the level sets $G_{\mathbf{k}}^{-1}(a)$ are orthogonal to the favorite directions of $\mathbf{k}$. Actually, it can be written out as a precise proposition.

Proposition 3.8. Let $\tau$ be a d-dimensional vector field defined on the level set $G_{\mathbf{k}}^{-1}(a)$ such that, at any point $t \in G_{\mathbf{k}}^{-1}(a), \tau(t)$ is orthogonal to $T_{t} G_{\mathbf{k}}^{-1}(a)$. Then, at any point $t$, the favorite directions of $\tau(t)$ are given by the favorite directions of k.

This formalizes an assertion in Longuet-Higgins (1957) Section 2.3, according to which the direction of the contour is near the principal direction. In this statement, the principal direction corresponds to our favorite direction.

Let us illustrate the notion of favorite direction on two-dimensional separable random wavevectors. We write $\mathbf{k}=\|\mathbf{k}\| u_{\Theta},\|\mathbf{k}\|$ and $\Theta$ being independent random variables. Note that for any $\varphi \in[0,2 \pi], \mathbf{k} \cdot u_{\varphi}=\|k\| \cos (\theta-\varphi)$.

On the one hand, if $\Theta$ is uniformly distributed on $[0,2 \pi]$, then $\mathbb{E}\left[\mathbf{k k}^{T}\right]=$ $\frac{1}{2} \mathbb{E}\left[\|k\|^{2}\right] I_{2}$, thus the set of favorite directions of $\mathbf{k}$ is $\mathbb{S}^{1}$. On the other hand, if $\Theta$ almost surely takes a fixed value $\theta_{0} \in[0,2 \pi]$ then the favorite directions of $\mathbf{k}$ are $\pm u_{\theta_{0}}$. Let us now focus on our favorite examples.

Example 1 (Toy model) If $\Theta$ admits a probability density function given by (2.5), for a given $\alpha>0$, then $\mathbb{E}\left[\mathbf{k k}^{T}\right]=\mathbb{E}\left[\|\mathbf{k}\|^{2}\right] \frac{1}{\alpha+2}\left(\begin{array}{cc}\alpha+1 & 0 \\ 0 & 1\end{array}\right)$. Hence, the favorite directions of $\mathbf{k}$ are $\pm u_{0}$.

Example 2 (Elementary model) If $\Theta$ is uniformly distributed on $[-h, h] \cup[\pi-$ $h, \pi+h]$ for some $0<h \leq \pi / 2$, then $\mathbb{E}\left[\mathbf{k k}^{T}\right]=\mathbb{E}\left[\|\mathbf{k}\|^{2}\right] \frac{1}{2}\left(\begin{array}{cc}1+\frac{\sin (2 h)}{2 h} & 0 \\ 0 & 1-\frac{\sin (2 h)}{2 h}\end{array}\right)$ and the favorite directions of $\mathbf{k}$ are again $\pm u_{0}$.

\section{Crest lines of planar Gaussian waves}

Let $\mathbf{k}$ be a two-dimensional random wavevector. We write $F$ its probability law and $G_{\mathbf{k}}$ its associated planar Gaussian random wave that we assume to be almost surely of class $\mathcal{C}^{3}$. We assume that $\mathbb{E}\left[\mathbf{k k}^{T}\right]$ is invertible, which equivalently excludes the case of $\mathbf{k}$ almost surely oriented along a fixed direction.

We fix a direction $\varphi \in[0, \pi)$ and we consider the crest line in direction $\varphi$. More precisely, we introduce the random set

$$
\left\{t \in \mathbb{R}^{2} ; G_{\mathbf{k}}^{\prime}(t) \cdot u_{\varphi}=0\right\},
$$

which contains all the points $t$ in $\mathbb{R}^{2}$ such that the gradient of $G_{\mathbf{k}}$ at point $t$ is orthogonal to direction $\varphi$. This set can be compared with the one of specular points in a fixed direction, as defined in Longuet-Higgins (1957). Crest lines intersected with nodal lines are also studied in Rudnick and Wigman $(2020+)$, in the case of 
arithmetic random waves, which are random eigenfunctions of the Laplacian on the two-dimensional torus.

4.1. Directional derivative as a random wave. The crest line can be considered as the nodal line of the random field $\left(G_{\mathbf{k}}^{\prime} \cdot u_{\varphi}\right)$, which is the partial derivative of $G_{\mathbf{k}}$ along vector $u_{\varphi}$. This random field is Gaussian, stationary, centred and we denote by $v_{0,0}(\varphi)$ its variance:

$$
v_{0,0}(\varphi)=\mathbb{E}\left[\left(G_{\mathbf{k}}^{\prime}(0) \cdot u_{\varphi}\right)^{2}\right]=u_{\varphi} \cdot \mathbb{E}\left[\mathbf{k k}^{T}\right] u_{\varphi}=\mathbb{E}\left[\left(\mathbf{k} \cdot u_{\varphi}\right)^{2}\right] .
$$

Note that $v_{0,0}(\varphi)$ does not vanish since $\mathbb{E}\left[\mathbf{k k}^{T}\right]$ is invertible.

By deriving the integral representation (2.1) of $r$, we obtain that the covariance function of $\left(G_{\mathbf{k}}^{\prime} \cdot u_{\varphi}\right)$ is

$$
\forall t \in \mathbb{R}^{2}, \quad \mathbb{E}\left[\left(G_{\mathbf{k}}^{\prime}(t) \cdot u_{\varphi}\right)\left(G_{\mathbf{k}}^{\prime}(0) \cdot u_{\varphi}\right)\right]=\int_{\mathbb{R}^{2}} \cos (t \cdot \lambda)\left(\lambda \cdot u_{\varphi}\right)^{2} d F(\lambda) .
$$

Therefore, the following lemma holds

Lemma 4.1. The covariance function of $\frac{G_{\mathbf{k}}^{\prime} \cdot u_{\varphi}}{v_{0,0}(\varphi)^{1 / 2}}$ is given by

$$
t \in \mathbb{R}^{2} \mapsto \mathbb{E}\left[\cos \left(\mathbf{K}_{\varphi} \cdot t\right)\right]
$$

where $\mathbf{K}_{\varphi}$ is a two-dimensional random vector whose probability distribution is equal to $\frac{\left(\lambda \cdot u_{\varphi}\right)^{2}}{v_{0,0}(\varphi)} d F(\lambda)$, with $F$ the probability measure of $\mathbf{k}$. In other words, $\mathbf{K}_{\varphi}$ is a random wavevector associated to $\frac{G_{\mathbf{k}}^{\prime} \cdot u_{\varphi}}{v_{0,0}(\varphi)^{1 / 2}}$.

We introduce the moments of measure $F$ and the ones of measure $\left(\lambda \cdot u_{\varphi}\right)^{2} d F(\lambda)$ : for any $(i, j) \in \mathbb{N}_{0}{ }^{2}$,

$$
\begin{aligned}
m_{i, j} & :=\int_{\mathbb{R}^{2}}\left(\lambda_{1}\right)^{i}\left(\lambda_{2}\right)^{j} d F(\lambda)=\mathbb{E}\left[\left(\mathbf{k} \cdot u_{0}\right)^{i}\left(\mathbf{k} \cdot u_{\pi / 2}\right)^{j}\right], \\
v_{i, j}(\varphi) & :=\int_{\mathbb{R}^{2}}\left(\lambda_{1}\right)^{i}\left(\lambda_{2}\right)^{j}\left(\lambda \cdot u_{\varphi}\right)^{2} d F(\lambda) .
\end{aligned}
$$

By a mere development, the moments $\left(v_{i, j}(\varphi)\right)$ can be expressed as polynomial functions of $(\cos \varphi, \sin \varphi)$, the coefficients depending linearly on the moments $\left(m_{i, j}\right)$ (see (A.2) in the Appendix section).

Until the end of this section and in the following one, we focus on the case of a separable random wavevector $\mathbf{k}$, that we write $\mathbf{k}=\|\mathbf{k}\| u_{\Theta}$. We denote by $\Xi$ the probability distribution of $\|\mathbf{k}\|$ on $(0,+\infty)$ and $\Lambda$ the one of $\Theta$ on $[0,2 \pi]$, assuming that $\|\mathbf{k}\|$ and $\Theta$ are independent. We introduce

$$
\forall j \in \mathbb{N}_{0}, \quad M_{j}=\mathbb{E}\left[\|\mathbf{k}\|^{j}\right]=\int_{\mathbb{R}^{+}} \rho^{j} d \Xi(\rho)
$$

the moments of the random variable $\|\mathbf{k}\|$ and $\mu_{j, k}$ the ones of $\Theta$, that is for any $(j, k) \in \mathbb{N}_{0}^{2}$,

$$
\mu_{i, j}=\int_{[0,2 \pi]} \cos ^{i} \theta \sin ^{j} \theta d \Lambda(\theta) .
$$

For any integrable function $h$,

$$
\mathbb{E}[h(\mathbf{k})]=\int_{[0,+\infty) \times[0,2 \pi]} h(\rho \cos \theta, \rho \sin \theta) d \Xi \otimes d \Lambda(\rho, \theta) .
$$


Therefore,

$$
\mathbb{E}\left[h\left(\mathbf{K}_{\varphi}\right)\right]=\frac{1}{v_{0,0}(\varphi)} \int_{(0,+\infty) \times[0,2 \pi]} h(\rho \cos \theta, \rho \sin \theta) \rho^{2} \cos ^{2}(\theta-\varphi) d \Xi \otimes d \Lambda(\rho, \theta) .
$$

To end with, we write

$$
\nu_{i, j}(\varphi)=\int_{0}^{2 \pi} \cos ^{i}(\theta) \sin ^{j}(\theta) \cos ^{2}(\theta-\varphi) d \Lambda(\theta) .
$$

We can directly state the following lemma.

Lemma 4.2. If $\mathbf{k}$ is separable, then $\mathbf{K}_{\varphi}$ is also separable and

$$
\forall(i, j) \in \mathbb{N}_{0}^{2}, \quad m_{i, j}=M_{i+j} \mu_{i, j} \quad \text { and } \quad v_{i, j}(\varphi)=M_{2+i+j} \nu_{i, j}(\varphi),
$$

assuming that the above moments exist.

4.2. Mean length of a crest line in a fixed direction. The Hausdorff dimension of a crest line is clearly equal to one. We consider its length within a compact domain $Q \subset \mathbb{R}^{2}$ such that $\mathcal{H}_{2}(Q)>0$,

$$
l(\mathbf{k}, Q, \varphi):=\mathcal{H}_{1}\left(\left\{t \in Q ; G_{\mathbf{k}}^{\prime}(t) \cdot u_{\varphi}=0\right\}\right) .
$$

Since the crest line of $G_{\mathbf{k}}$ in direction $\varphi$ is nothing but the nodal line of $\frac{G_{\mathbf{k}}^{\prime} \cdot u_{\varphi}}{v_{0,0}(\varphi)^{1 / 2}}$, Lemma 4.1 allows us to apply Proposition 3.4 with the random wavevector $\mathbf{K}_{\varphi}$. It yields the following proposition.

Proposition 4.3. We assume that $\mathbf{k}$ is separable and that $\mathbb{E}\left[\mathbf{k k}^{T}\right]$ is invertible. Then for any $\varphi \in[0, \pi)$ such that $\mathbb{E}\left[\mathbf{K}_{\varphi} \mathbf{K}_{\varphi}^{T}\right]$ is invertible,

$$
\mathbb{E}[l(\mathbf{k}, Q, \varphi)]=\mathcal{H}_{2}(Q) \frac{1}{\pi \sqrt{2}} \mathbb{E}\left[\left\|\mathbf{K}_{\varphi}\right\|^{2}\right]^{1 / 2} \mathcal{F}\left(c\left(\widetilde{\mathbf{K}}_{\varphi}\right)\right),
$$

where function $\mathcal{F}$ has been introduced in Proposition 3.4,

$$
\begin{aligned}
\mathbb{E}\left(\left\|\mathbf{K}_{\varphi}\right\|^{2}\right) & =\frac{M_{4}}{M_{2}}, \\
c\left(\widetilde{\mathbf{K}}_{\varphi}\right) & =\frac{\left(\left(\nu_{2,0}(\varphi)-\nu_{0,2}(\varphi)\right)^{2}+4 \nu_{1,1}(\varphi)^{2}\right)^{1 / 2}}{\nu_{0,0}(\varphi)} .
\end{aligned}
$$

We refer to the Appendix Section A.3 for the proof of (4.4) and (4.5). See also Formula (A.2) in that section to get an expression of $c\left(\widetilde{\mathbf{K}}_{\varphi}\right)$ in terms of the fourth-order moments $\left(m_{i, j}\right)_{(i, j) \in \mathbb{N}_{0}{ }^{2}, i+j=4}$.

According to Proposition $4.3, \mathbb{E}[l(\mathbf{k}, Q, \varphi)]$ depends on the directional distribution of $\mathbf{k}$ through the factor $\mathcal{F}\left(c\left(\widetilde{\mathbf{K}}_{\varphi}\right)\right.$ ) (involving the fourth-order moments of $\mathbf{k}$ ), and on its radial distribution through the factor $\mathbb{E}\left[\left\|\mathbf{K}_{\varphi}\right\|^{2}\right]$ (involving the secondorder and the fourth-order moment of $\Xi$ ). Consequently, the mean length of crest lines is a decreasing function of the anisotropy of $\mathbf{K}_{\varphi}$. The variations of the expected length of the crests with respect to the direction can be studied through the variations of the map $\varphi \mapsto c\left(\widetilde{\mathbf{K}}_{\varphi}\right)$. For more detailed results, see the Appendix Section A.4.

Let us remark that a similar formula is also derived in Azaïs and Wschebor (2009, Proposition 11.4) and in Azaiis et al. (2005, Assertion 3). It is apparently different from ours, since the gradient of the random field $G_{\mathbf{k}}^{\prime} \cdot u_{\varphi}$ is not computed according 
to the directions of the canonical basis but according to the ones of $\left(u_{\varphi}, u_{\varphi+\pi / 2}\right)$. Nevertheless they actually coincide.

Now we apply Proposition 4.3 to our separable examples. All is about computing the coherency index of $\widetilde{\mathbf{K}}_{\varphi}$. For each case, we examine the crest direction(s) that maximize(s) the expectation of the crest length. In Longuet-Higgins (1957) or in Azaiis et al. (2005), a rule of thumb is suggested claiming that the direction [that maximises the expected length of crests] is orthogonal to the direction for the maximum integral of the spectrum, i.e. is the most probable direction for the waves. In this statement, the "most probable direction for the waves" refers to the mode of the random wavevector $\widetilde{\mathbf{k}}$. However, according to our examples, such a rule is not necessarily satisfied. This can be explained by the expectation formula of Proposition 4.3 itself, which shows a dependency on both the second-order and the fourth-order moments of $\mathbf{k}$, and not on the mode of $\widetilde{\mathbf{k}}$.

Examples We consider a random wavevector $\mathbf{k}$ that we write $\mathbf{k}=\|\mathbf{k}\| u_{\Theta}$ as before and we use the formulas of Appendix Sections A.3 and A.4.

- Toy Model. The probability density function of $\Theta$ is given by (2.5), for some fixed $\alpha>0$ and $\theta_{0}$ equal to zero. The mode of $\widetilde{\mathbf{k}}$ is 0 in that case. An asymptotic expansion of $\varphi \mapsto c\left(\widetilde{\mathbf{K}}_{\varphi}\right)^{2}$ near $\varphi=\pi / 2$ is performed in Lemma A.5 in the Appendix section. It shows that the expected length of crests admits a local maximum at $\varphi=\pi / 2$, which is precisely orthogonal to the most probable direction of $\mathbf{k}$ and to its favorite direction as well.

- Elementary wave. We assume that $\Theta$ is uniformly distributed on $[-h, h] \cup$ $[\pi-h, \pi+h]$. The moments of $\widetilde{\mathbf{K}}_{\varphi}$ can be computed thanks to Formula (A.3) and Lemma A.2. Figure A.2 shows the graph of $\varphi \mapsto c\left(\widetilde{\mathbf{K}}_{\varphi}\right)^{2}$. The expected length of crests appears to admit a maximum at $\varphi=\pi / 2$, thus it is orthogonal to the favorite direction of $\mathbf{k}$.

- A very special example. Let $\frac{1}{4} \sum_{j=0}^{3} \delta_{j \pi / 2}$ be the distribution of $\Theta$ on $[0,2 \pi], \delta_{a}$ being the Dirac measure at point $a$. It is called Cilleruelo measure in Rudnick and Wigman $(2020+)$. The modes of $\widetilde{\mathbf{k}}$ are $\left\{u_{j \pi / 2}\right.$ : $j=0,1,2,3\}$. We can mention that it does not admit any favorite direction. Computing the moments of $F$, we get $m_{1,1}=m_{2,2}=m_{3,1}=m_{3,1}=0$ whereas $m_{2,0}=m_{0,2}=\frac{M_{2}}{2}$ and $m_{4,0}=m_{0,4}=\frac{M_{4}}{2}$. Since

$$
\mathbb{E}\left[\widetilde{\mathbf{K}}_{\varphi} \widetilde{\mathbf{K}}_{\varphi}^{T}\right]=\left(\begin{array}{cc}
\cos ^{2} \varphi & 0 \\
0 & \sin ^{2} \varphi
\end{array}\right),
$$

$c\left(\widetilde{\mathbf{K}}_{\varphi}\right)=|\cos (2 \varphi)|$. Since $\mathcal{F}$ is strictly decreasing on $[0,1]$, the mean length of crests is maximal when $\cos (2 \varphi)=0$, i.e. for $\varphi=\pi / 4$ or $3 \pi / 4$ modulo $\pi$. These directions are not orthogonal to the modes of $\widetilde{\mathbf{k}}$.

\section{Appendix A. Appendix}

A.1. Moments of two specific planar random wavevectors. Let $\mathbf{k}$ be a planar separable wavevector that we write $\mathbf{k}=\|\mathbf{k}\| \widetilde{\mathbf{k}}$ with $\|\mathbf{k}\|$ and $\widetilde{\mathbf{k}}$ independent and $\widetilde{\mathbf{k}}=(\cos \Theta, \sin \Theta)$. In the two following lemmas, we compute the moments of $\widetilde{\mathbf{k}}$, i.e.

$$
\mu_{j, k}=\mathbb{E}\left[(\cos \Theta)^{j}(\sin \Theta)^{k}\right]
$$


for any non negative integers $j$ and $k$, in two specific cases, namely the toy model and the elementary model as introduced in Section 2.4.2.

Lemma A.1. If $\Theta$ has a probability density function given by

$$
\theta \mapsto C_{\alpha}|\cos \theta|^{\alpha} \quad \text { with } \quad C_{\alpha}=\frac{\Gamma(1+\alpha / 2)}{2 \sqrt{\pi} \Gamma(1 / 2+\alpha / 2)}
$$

for some nonnegative constant $\alpha$, then the following formulas hold:

- $\mu_{0,0}=1$

- $\mu_{j, k}=0$ whenever $j$ or $k$ is odd

- $\mu_{j, 0}=\frac{C_{\alpha}}{C_{\alpha+j}}=\frac{(\alpha+1)(\alpha+3) \cdots(\alpha+j-1)}{(\alpha+2)(\alpha+4) \cdots(\alpha+j)}$ for $j$ even $\geq 2$

- for any even integers $j$ and $k, \mu_{j, k}=\sum_{i=0}^{k / 2}(-1)^{i}\left(\begin{array}{c}k / 2 \\ i\end{array}\right) \mu_{j+2 i, 0}$.

In particular, it yields the non-zero second and fourth-order moments of $\widetilde{\mathbf{k}}$ :

$$
\begin{aligned}
& \mu_{2,0}=\frac{\alpha+1}{\alpha+2} ; \mu_{0,2}=\frac{1}{\alpha+2} \text { and hence } \mathbb{E}\left[\widetilde{\mathbf{k}} \widetilde{\mathbf{k}}^{T}\right]=\frac{1}{\alpha+2}\left(\begin{array}{cc}
\alpha+1 & 0 \\
0 & 1
\end{array}\right) \\
& \mu_{4,0}=\frac{(\alpha+1)(\alpha+3)}{(\alpha+2)(\alpha+4)} ; \mu_{0,4}=\frac{3}{(\alpha+2)(\alpha+4)} ; \mu_{2,2}=\frac{\alpha+1}{(\alpha+2)(\alpha+4)}
\end{aligned}
$$

Proof: It is clear that $\mu_{0,0}=1, \mu_{j, k}=0$ whenever $j$ or $k$ is odd and that $\mu_{j, 0}=$ $C_{\alpha} / C_{\alpha+j}$ for any even integer $j$. Using the explicit value of $C_{\alpha}$ yields the value of $\mu_{j, 0}$. Finally, for any even integers $j$ and $k$, writing $\sin ^{2} \theta=1-\cos ^{2} \theta$ yields the formula for $\mu_{j, k}$.

Lemma A.2. If $\Theta$ is uniformly distributed on $[-h, h] \cup[\pi-h, \pi+h]$ for some constant $h \in(0, \pi / 2)$, then the following formulas hold:

- $\mu_{0,0}=1$,

- $\mu_{j, k}=0$ whenever $j$ or $k$ is odd,

- $\mu_{2,0}=\frac{1}{2}(1+\operatorname{sinc}(2 h)) ; \mu_{0,2}=\frac{1}{2}(1-\operatorname{sinc}(2 h)) ; \mu_{2,2}=\frac{1}{8}(1-\operatorname{sinc}(4 h))$,

- $\mu_{4,0}=\frac{1}{8}(3+4 \operatorname{sinc}(2 h)+\operatorname{sinc}(4 h)) ; \mu_{0,4}=\frac{1}{8}(3-4 \operatorname{sinc}(2 h)+\operatorname{sinc}(4 h))$,

where $\operatorname{sinc}(\theta)=\frac{\sin (\theta)}{\theta}$ for any $\theta \neq 0$. In particular, this implies

$$
\mathbb{E}\left[\widetilde{\mathbf{k}} \widetilde{\mathbf{k}}^{T}\right]=\frac{1}{2}\left(\begin{array}{cc}
1+\operatorname{sinc}(2 h) & 0 \\
0 & 1-\operatorname{sinc}(2 h)
\end{array}\right) .
$$

Proof: Symmetry arguments explain the vanishing moments, while the non-zero ones can be evaluated by linearizing trigonometric functions.

\section{A.2. Variations of map $\mathcal{F}$.}

Lemma A.3. Let $\mathcal{E}$ be the elliptic integral given by $\mathcal{E}(x)=\int_{0}^{\pi / 2}\left(1-x^{2} \sin ^{2} \theta\right)^{1 / 2} d \theta$, for $x \in[0,1]$. Then, the map $\mathcal{F}: c \mapsto(1+c)^{1 / 2} \mathcal{E}\left(\left(\frac{2 c}{1+c}\right)^{1 / 2}\right)$ is strictly decreasing on $[0,1]$. 
Proof: For any $k \in[0,1), \mathcal{E}^{\prime}(k)=-k \int_{0}^{\pi / 2} \frac{\sin ^{2} \theta}{\left(1-k^{2} \sin ^{2} \theta\right)^{1 / 2}} d \theta$. Therefore, for any $c \in[0,1)$,

$$
\begin{aligned}
\mathcal{F}^{\prime}(c) & =\frac{1}{2}(1+c)^{-1 / 2} \mathcal{E}\left(\left(\frac{2 c}{1+c}\right)^{1 / 2}\right)+(1+c)^{1 / 2} \frac{(2 c)^{-1 / 2}}{(1+c)^{3 / 2}} \mathcal{E}^{\prime}\left(\left(\frac{2 c}{1+c}\right)^{1 / 2}\right) \\
& =\frac{1}{2}(1+c)^{-1 / 2} \int_{0}^{\pi / 2}\left[\left(1-\frac{2 c}{1+c} \sin ^{2} \theta\right)^{1 / 2}-\frac{\frac{2}{1+c} \sin ^{2} \theta}{\left(1-\frac{2 c}{1+c} \sin ^{2} \theta\right)^{1 / 2}}\right] d \theta \\
& =\frac{1}{2}(1+c)^{-1 / 2} \int_{0}^{\pi / 2} \frac{\cos (2 \theta)}{\left(1-\frac{2 c}{1+c} \sin ^{2} \theta\right)^{1 / 2}} d \theta .
\end{aligned}
$$

It remains to show that the above integral, which we call $J(k)$ with $k=\left(\frac{2 c}{1+c}\right)^{1 / 2}$, is negative. Splitting the integral $J(k):=\int_{0}^{\pi / 2} \frac{\cos (2 \theta)}{\left(1-k^{2} \sin ^{2} \theta\right)^{1 / 2}} d \theta$ into two parts, on $[0, \pi / 4]$ and on $[\pi / 4, \pi / 2]$, and performing the change of variables $\theta^{\prime}=\pi / 2-\theta$ within the second part, we get

$$
J(k)=\int_{0}^{\pi / 4} \cos (2 \theta)\left[\frac{1}{\left(1-k^{2} \sin ^{2} \theta\right)^{1 / 2}}-\frac{1}{\left(1-k^{2} \cos ^{2} \theta\right)^{1 / 2}}\right] d \theta,
$$

which is negative since $\cos \theta>\sin \theta$ for $\theta \in(0, \pi / 4)$.

A.3. Coherency index of $\mathbf{K}_{\varphi}$. In this section, we prove Formulas (4.4) and (4.5) that are stated in Proposition 4.3. We start with a general result that allows us to compute the coherency index of $\mathbf{K}_{\varphi}$.

Lemma A.4. (1) $\mathbb{E}\left[\mathbf{K}_{\varphi} \mathbf{K}_{\varphi}^{T}\right]=\frac{1}{v_{0,0}(\varphi)} \mathbb{V}\left[G_{\mathbf{k}}^{\prime \prime}(0) u_{\varphi}\right]$, where

$$
\begin{array}{r}
v_{0,0}(\varphi)=m_{2,0} \cos ^{2} \varphi+2 m_{1,1} \cos \varphi \sin \varphi+m_{0,2} \sin ^{2} \varphi \\
\text { and } \mathbb{V}\left[G_{\mathbf{k}}^{\prime \prime}(0) u_{\varphi}\right]=\left(\begin{array}{ll}
v_{2,0}(\varphi) & v_{1,1}(\varphi) \\
v_{1,1}(\varphi) & v_{0,2}(\varphi)
\end{array}\right) \\
\text { with }\left\{\begin{array}{l}
v_{2,0}(\varphi)=\cos ^{2} \varphi m_{4,0}+2 \cos \varphi \sin \varphi m_{3,1}+\sin ^{2} \varphi m_{2,2}, \\
v_{0,2}(\varphi)=\cos ^{2} \varphi m_{2,2}+2 \cos \varphi \sin \varphi m_{1,3}+\sin ^{2} \varphi m_{0,4}, \\
v_{1,1}(\varphi)=\cos ^{2} \varphi m_{3,1}+2 \cos \varphi \sin \varphi m_{2,2}+\sin ^{2} \varphi m_{1,3} .
\end{array}\right. \\
\text { (2) } \mathbb{E}\left[\left\|\mathbf{K}_{\varphi}\right\|^{2}\right]=\frac{v_{2,0}(\varphi)+v_{0,2}(\varphi)}{v_{0,0}(\varphi)} \\
\text { (3) } c\left(\mathbf{K}_{\varphi}\right)=\frac{\left(\left(v_{2,0}(\varphi)-v_{0,2}(\varphi)\right)^{2}+4 v_{1,1}(\varphi)^{2}\right)^{1 / 2}}{v_{2,0}(\varphi)+v_{0,2}(\varphi)}
\end{array}
$$

Proof: As a consequence of Lemma 4.1, $\mathbb{E}\left[\mathbf{K}_{\varphi} \mathbf{K}_{\varphi}^{T}\right]$ is the covariance matrix of the gradient vector of $\frac{G_{\mathbf{k}}^{\prime} \cdot u_{\varphi}}{v_{0,0}(\varphi)^{1 / 2}}$. But the gradient vector of $G_{\mathbf{k}}^{\prime} \cdot u_{\varphi}$ is actually the vectorial random field $\left(G_{\mathbf{k}}^{\prime \prime}(t) u_{\varphi}\right)_{t \in \mathbb{R}^{2}}$, with covariance matrix $\mathbb{V}\left[G_{\mathbf{k}}^{\prime \prime}(0) u_{\varphi}\right]$. It proves Point 1.

Point 2 stems from the equality $\mathbb{E}\left[\left\|\mathbf{K}_{\varphi}\right\|^{2}\right]=\operatorname{Trace}\left(\mathbb{E}\left[\mathbf{K}_{\varphi} \mathbf{K}_{\varphi}^{T}\right]\right)$ and Point 3 derives from Formula (2.3).

Now, let us assume that $\mathbf{k}$ is separable. Lemma 4.1 ensures that $\mathbf{K}_{\varphi}$ is also separable. Then

$$
\mathbb{E}\left[\widetilde{\mathbf{K}}_{\varphi} \widetilde{\mathbf{K}}_{\varphi}^{T}\right]=\frac{1}{\nu_{0,0}(\varphi)}\left(\begin{array}{ll}
\nu_{2,0}(\varphi) & \nu_{1,1}(\varphi) \\
\nu_{1,1}(\varphi) & \nu_{0,2}(\varphi)
\end{array}\right)
$$


where the moments $\left(\nu_{i, j}(\varphi)\right)$ have been defined by (4.3). It results from Lemma 4.2 and from $\nu_{2,0}(\varphi)+\nu_{0,2}(\varphi)=\nu_{0,0}(\varphi)$ that $\mathbb{E}\left[\left\|\mathbf{K}_{\varphi}\right\|^{2}\right]=\frac{M_{4}}{M_{2}}$ (which is actually equation (4.4)) and that the trace of $\mathbb{E}\left[\widetilde{\mathbf{K}}_{\varphi} \widetilde{\mathbf{K}}_{\varphi}^{T}\right]$ equals $\mathbb{E}\left[\left\|\widetilde{\mathbf{K}}_{\varphi}\right\|^{2}\right]=1$ (this can also be seen as a consequence of $\left.\widetilde{\mathbf{K}}_{\varphi} \in \mathbb{S}^{1}\right)$. Consequently, its coherency index is

$$
c\left(\widetilde{\mathbf{K}}_{\varphi}\right)=\frac{\left(\left(\nu_{2,0}(\varphi)-\nu_{0,2}(\varphi)\right)^{2}+4 \nu_{1,1}(\varphi)^{2}\right)^{1 / 2}}{\nu_{0,0}(\varphi)},
$$

which is actually equation (4.5).

A.4. Variations of the mean length of crests. Thanks to Proposition 4.3, we know that the mean length of a crest of direction $\varphi$ is given as a decreasing function of $c\left(\widetilde{\mathbf{K}}_{\varphi}\right)$. Recall that Formula (4.5) allows us to write out $c\left(\widetilde{\mathbf{K}}_{\varphi}\right)^{2}$ as

$$
c\left(\widetilde{\mathbf{K}}_{\varphi}\right)^{2}=\frac{1}{\nu_{0,0}(\varphi)^{2}}\left(\left(\nu_{2,0}(\varphi)-\nu_{0,2}(\varphi)\right)^{2}+4 \nu_{1,1}(\varphi)^{2}\right),
$$

where the $\nu_{i, j}(\varphi)$ 's are defined by (4.3). By Lemma 4.2, the moments $\nu_{i, j}(\varphi)$ are such that $\nu_{i, j}(\varphi)=\frac{1}{M_{2+i+j}} v_{i, j}(\varphi)$. We use (A.2) to express $v_{i, j}(\varphi)$ in terms of the moments of $\mathbf{k}$ denoted by $m_{i, j}$. Since $m_{i, j}=M_{i+j} \mu_{i, j}$, Lemma 4.2 also yields

$$
\left\{\begin{array}{l}
\nu_{0,0}(\varphi)=\mu_{2,0} \cos ^{2} \varphi+2 \mu_{1,1} \cos \varphi \sin \varphi+\mu_{0,2} \sin ^{2} \varphi \\
\nu_{2,0}(\varphi)=\mu_{4,0} \cos ^{2} \varphi+2 \mu_{3,1} \cos \varphi \sin \varphi+\mu_{2,2} \sin ^{2} \varphi \\
\nu_{0,2}(\varphi)=\mu_{2,2} \cos ^{2} \varphi+2 \mu_{1,3} \cos \varphi \sin \varphi+\mu_{0,4} \sin ^{2} \varphi \\
\nu_{1,1}(\varphi)=\mu_{3,1} \cos ^{2} \varphi+2 \mu_{2,2} \cos \varphi \sin \varphi+\mu_{1,3} \sin ^{2} \varphi .
\end{array}\right.
$$

In the case where the moments $\mu_{1,1}, \mu_{1,3}$ and $\mu_{3,1}$ simultaneously vanish, which is the case in our two favorite examples (see Lemmas A.1 and A.2), we like to remark that there exist two polynomials of degree two, say $P$ and $Q$, such that

$$
\forall \varphi \in[0,2 \pi], \quad c\left(\widetilde{\mathbf{K}}_{\varphi}\right)^{2}=\frac{P(\cos (2 \varphi))}{Q(\cos (2 \varphi))} .
$$

Consequently, $\varphi=0$ and $\varphi=\pi / 2$ appear as stationary points of the $\pi$-periodic map $\varphi \mapsto c\left(\widetilde{\mathbf{K}}_{\varphi}\right)^{2}$, whose graph is symmetric with respect to $\pi / 2$.

A.4.1. The toy model case. We assume that the direction of $\mathbf{k}$ is given by the toy model (see Example 1 in Section 2.4.2). Using (A.3) and the expression for the $\mu_{i, j}$ 's given by Lemma A.1, we can compute $c\left(\widetilde{\mathbf{K}}_{\varphi}\right)^{2}$. Figure A.1 shows the graph of $\varphi \mapsto c\left(\widetilde{\mathbf{K}}_{\varphi}\right)^{2}$ for various values of $\alpha$. We observe that $\varphi \mapsto c\left(\widetilde{\mathbf{K}}_{\varphi}\right)^{2}$ is minimal at $\varphi=\pi / 2$, which is substantiated by the next lemma.

Lemma A.5. The map $\varphi \mapsto c\left(\widetilde{\mathbf{K}}_{\varphi}\right)$ admits a local minimum at $\varphi=\pi / 2$.

Proof: We will perform an asymptotic expansion of $c\left(\widetilde{\mathbf{K}}_{\pi / 2+\varphi}\right)^{2}$ near $\varphi=0$ in order to show that $\varphi \mapsto c\left(\widetilde{\mathbf{K}}_{\varphi}\right)$ admits a local minimum at $\pi / 2$.

In the following lines, we write $g(\varphi)=\mathcal{O}(h(\varphi))$ as $\varphi$ tends to 0 , if there exists $\varphi_{0} \in(0,2 \pi)$ and $M>0$ such that

$$
\forall \varphi \in[0,2 \pi],|\varphi|<\left|\varphi_{0}\right| \Rightarrow|g(\varphi)| \leq M|h(\varphi)| .
$$




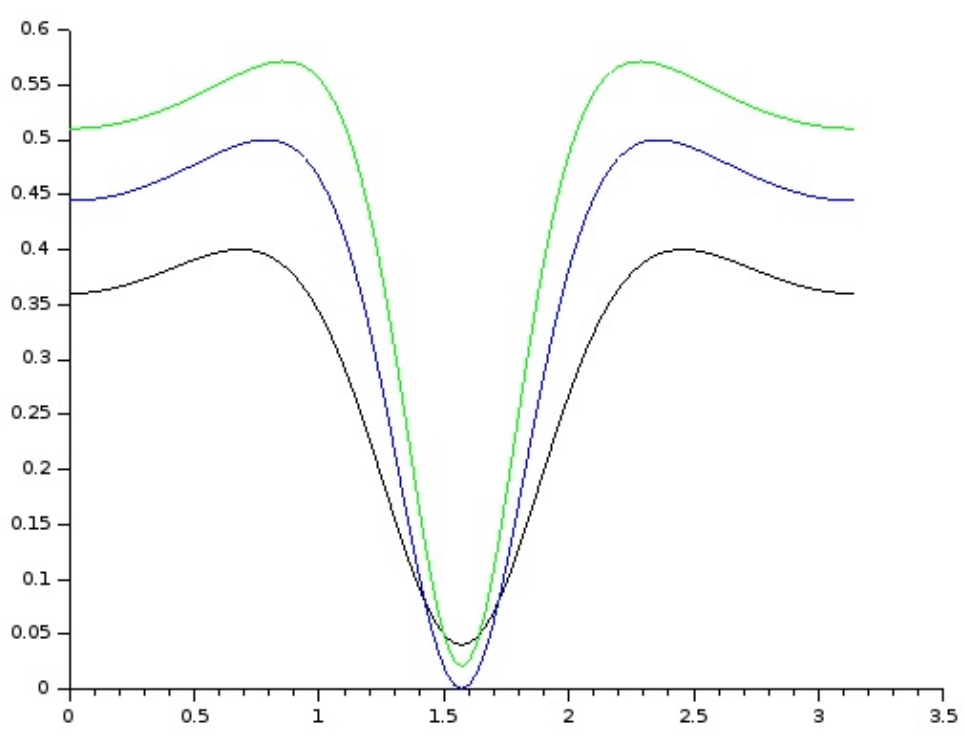

Figure A.1. Graph of $\varphi \mapsto c\left(\widetilde{\mathbf{K}}_{\varphi}\right)^{2}$ for $\alpha=1$ (black), $\alpha=2$ (blue) and $\alpha=3$ (green).

In particular, we write $\left\{\begin{array}{l}\cos (\pi / 2+\varphi)=-\varphi+\frac{\varphi^{3}}{6}+\mathcal{O}\left(\varphi^{4}\right), \\ \sin (\pi / 2+\varphi)=1-\frac{\varphi^{2}}{2}+\mathcal{O}\left(\varphi^{4}\right) .\end{array}\right.$

From (A.3) and Lemma A.1, we get

$$
\begin{aligned}
\nu_{0,0}(\pi / 2+\varphi) & =\mu_{0,2}+\left(\mu_{2,0}-\mu_{0,2}\right) \varphi^{2}+\mathcal{O}\left(\varphi^{4}\right) \\
& =\frac{1}{\alpha+2}\left(1+\alpha \varphi^{2}\right)+\mathcal{O}\left(\varphi^{4}\right) \\
\nu_{2,0}(\pi / 2+\varphi) & =\mu_{2,2}+\left(\mu_{4,0}-\mu_{2,2}\right) \varphi^{2}+\mathcal{O}\left(\varphi^{4}\right) \\
& =\frac{\alpha+1}{(\alpha+2)(\alpha+4)}\left(1+(\alpha+2) \varphi^{2}\right)+\mathcal{O}\left(\varphi^{4}\right) \\
\nu_{0,2}(\pi / 2+\varphi) & =\mu_{0,4}+\left(\mu_{2,2}-\mu_{0,4}\right) \varphi^{2}+\mathcal{O}\left(\varphi^{4}\right) \\
& =\frac{1}{(\alpha+2)(\alpha+4)}\left(3+(\alpha-2) \varphi^{2}\right)+\mathcal{O}\left(\varphi^{4}\right) \\
\nu_{1,1}(\pi / 2+\varphi) & =2 \mu_{2,2} \varphi\left(1-\frac{1}{2} \varphi^{2}\right)+\mathcal{O}\left(\varphi^{4}\right) \\
& =\frac{\alpha+1}{(\alpha+2)(\alpha+4)} \varphi\left(2-\varphi^{2}\right)+\mathcal{O}\left(\varphi^{4}\right) .
\end{aligned}
$$

After some algebra, it gives

$$
c\left(\widetilde{\mathbf{K}}_{\pi / 2+\varphi}\right)^{2}=(\alpha-2)^{2}+24 \alpha(\alpha+1) \varphi^{2}+\mathcal{O}\left(\varphi^{4}\right),
$$


which clearly admits a minimum at $\varphi=0$.

A.4.2. The elementary model case. We assume that the direction of $\mathbf{k}$ is given by the elementary model (see Example 2 in Section 2.4.2). Using (A.3) and the expression for the $\mu_{i, j}$ 's given by Lemma A.2, one can compute $c\left(\widetilde{\mathbf{K}}_{\varphi}\right)^{2}$. Figure A.2 shows the graph of $\varphi \mapsto c\left(\widetilde{\mathbf{K}}_{\varphi}\right)^{2}$ for various values of $h$. We again observe that it is minimal at $\varphi=\pi / 2$.

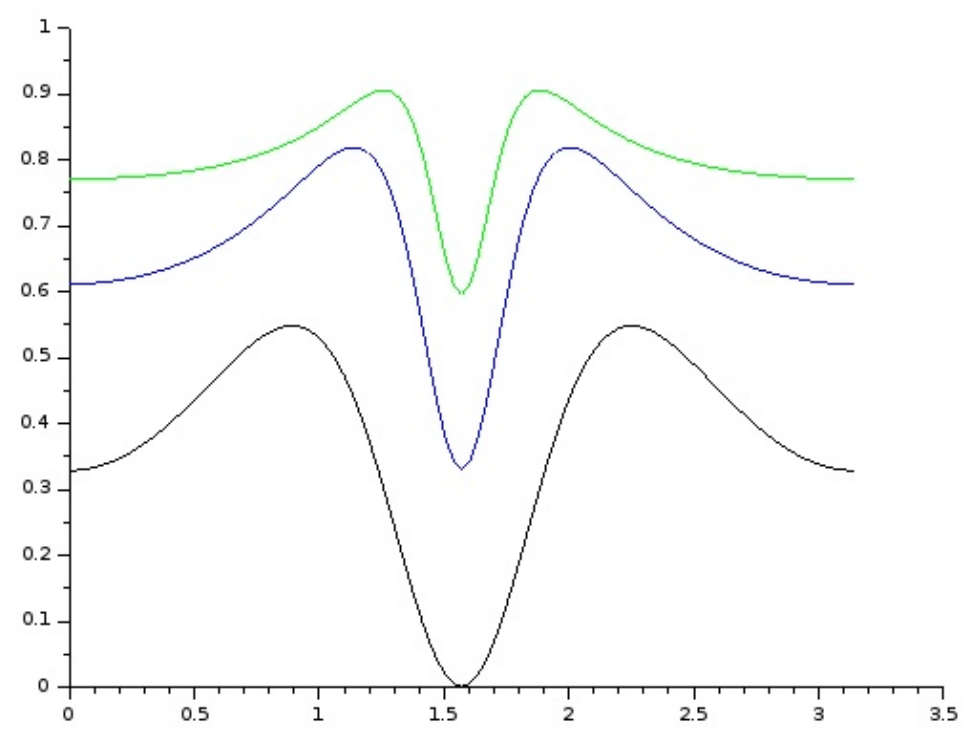

Figure A.2. Graph of $\varphi \mapsto c\left(\widetilde{\mathbf{K}}_{\varphi}\right)^{2}$ for $h=\pi / 3$ (black), $h=\pi / 5$ (blue) and $h=\pi / 7$ (green).

\section{References}

Aberg, S., Rychlik, I., and Leadbetter, M. R. Palm distributions of wave characteristics in encountering seas. Ann. Appl. Probab., 18 (3), 1059-1084 (2008). MR2418238.

Ade, P. A. R., Aghanim, N., Akrami, Y., Aluri, P. K., Arnaud, M., Ashdown, M., Aumont, J., et al. Planck 2015 results - XVI. Isotropy and statistics of the CMB. Astronomy and Astrophysics, 594, A16 (2016). DOI: 10.1051/0004$6361 / 201526681$.

Adler, R. J. and Taylor, J. E. Random fields and geometry. Springer Monographs in Mathematics. Springer, New York (2007). ISBN 978-0-387-48112-8. MR2319516. Allard, D., Senoussi, R., and Porcu, E. Anisotropy models for spatial data. Math. Geosci., 48 (3), 305-328 (2016). MR3476704. 
Azaïs, J.-M., León, J. R., and Ortega, J. Geometrical characteristics of Gaussian sea waves. J. Appl. Probab., 42 (2), 407-425 (2005). MR2145485.

Azaïs, J.-M., León, J. R., and Wschebor, M. Rice formulae and Gaussian waves. Bernoulli, 17 (1), 170-193 (2011). MR2797987.

Azaïs, J.-M. and Wschebor, M. Level sets and extrema of random processes and fields. John Wiley \& Sons, Inc., Hoboken, NJ (2009). ISBN 978-0-470-40933-6. MR2478201.

Beliaev, D., Cammarota, V., and Wigman, I. Two point function for critical points of a random plane wave. Int. Math. Res. Not. IMRN, (9), 2661-2689 (2019). MR3947635.

Berry, M. V. Regular and irregular semiclassical wavefunctions. J. Phys. A, 10 (12), 2083-2091 (1977). MR489542.

Berry, M. V. Statistics of nodal lines and points in chaotic quantum billiards: perimeter corrections, fluctuations, curvature. J. Phys. A, 35 (13), 3025-3038 (2002). MR1913853.

Berry, M. V. and Dennis, M. R. Phase singularities in isotropic random waves. $R$. Soc. Lond. Proc. Ser. A Math. Phys. Eng. Sci., 456 (2001), 2059-2079 (2000). MR1794716.

Biermé, H., Moisan, L., and Richard, F. A turning-band method for the simulation of anisotropic fractional Brownian fields. J. Comput. Graph. Statist., 24 (3), 885-904 (2015). MR3397238.

Bonami, A. and Estrade, A. Anisotropic analysis of some Gaussian models. J. Fourier Anal. Appl., 9 (3), 215-236 (2003). MR1988750.

De Angelis, L., Alpeggiani, F., Di Falco, A., and Kuipers, L. Spatial Distribution of Phase Singularities in Optical Random Vector Waves. Phys. Rev. Lett., 117, 093901 (2016). DOI: 10.1103/PhysRevLett.117.0939014.

Fournier, J. Identification and isotropy characterization of deformed random fields through excursion sets. Adv. in Appl. Probab., 50 (3), 706-725 (2018). MR3877250.

Klatt, M. Morphometry of random spatial structures in physics. Ph.D. thesis, UFAU Forschungen, Reihe B, Medizin, Naturwissenschaft, Technika (2016).

Krishnapur, M., Kurlberg, P., and Wigman, I. Nodal length fluctuations for arithmetic random waves. Ann. of Math. (2), 177 (2), 699-737 (2013). MR3010810.

Kurlberg, P. and Wigman, I. On probability measures arising from lattice points on circles. Math. Ann., 367 (3-4), 1057-1098 (2017). MR3623219.

Lindgren, G. Slepian models for the stochastic shape of individual Lagrange sea waves. Adv. in Appl. Probab., 38 (2), 430-450 (2006). MR2264951.

Longuet-Higgins, M. S. The statistical analysis of a random, moving surface. Philos. Trans. Roy. Soc. London Ser. A, 249, 321-387 (1957). MR87257.

Mardia, K. V. and Jupp, P. E. Directional statistics. Wiley Series in Probability and Statistics. John Wiley \& Sons, Ltd., Chichester (2000). ISBN 0-471-95333-4. MR1828667.

Marinucci, D., Peccati, G., Rossi, M., and Wigman, I. Non-universality of nodal length distribution for arithmetic random waves. Geom. Funct. Anal., 26 (3), 926-960 (2016). MR3540457.

Nourdin, I., Peccati, G., and Rossi, M. Nodal statistics of planar random waves. Comm. Math. Phys., 369 (1), 99-151 (2019). MR3959555. 
Olhede, S. C., Ramírez, D., and Schreier, P. J. Detecting directionality in random fields using the monogenic signal. IEEE Trans. Inform. Theory, 60 (10), 64916510 (2014). MR3265036.

Polisano, K., Clausel, M., Perrier, V., and Condat, L. Riesz-based orientation of localizable Gaussian fields $(2020+)$. To appear in Applied and Computational Harmonic Analysis.

Richard, F. and Biermé, H. Statistical Tests of Anisotropy for Fractional Brownian Textures. Application to Full-field Digital Mammography. Journal of Mathematical Imaging and Vision, 36 (3), 227-240 (2010). DOI: 10.1007/s10851-009-0181-y.

Rudnick, Z. and Wigman, I. On the volume of nodal sets for eigenfunctions of the Laplacian on the torus. Ann. Henri Poincaré, 9 (1), 109-130 (2008). MR2389892.

Rudnick, Z. and Wigman, I. Points on nodal lines with given direction $(2020+)$. To appear in J. of Spectral Theory. 